\title{
The MISO Interference Channel as a Model for Non-Orthogonal Spectrum Sharing
}

Johannes Lindblom

\section{SOMmunication SิŶSTEMS}

Division of Communication Systems Department of Electrical Engineering (ISY)

Linköping University, SE-581 83 Linköping, Sweden www.commsys.isy.liu.se

Linköping 2013 
The MISO Interference Channel as a Model for Non-Orthogonal Spectrum Sharing

(c) 2013 Johannes Lindblom, unless otherwise noted.

ISBN 978-91-7519-478-3

ISSN 0345-7524

Printed in Sweden by LiU-Tryck, Linköping 2013 
"There are no shortcuts to any place worth going."

Beverly Sills 



\section{Abstract}

The demand for wireless communications services has increased during the last decades. To meet this demand, there is a need for allocating larger frequency bands. However, most of the frequency bands (or spectrum) suitable for wireless communication are occupied and allocated to licensed systems. Long-term (order of years) contracts enforce the operators to use separate bands. Also, within an operator, neighboring cells have used separate frequency bands to avoid causing interference to each others' mobile users. The drawback of such operation is low spectral efficiency due to unused spectrum and low flexibility in the allocation of resources for the mobile users. To overcome these problems, so-called spectrum sharing has been proposed. The idea is that different operators (inter-operator spectrum sharing) or neighboring cells (intra-operator spectrum sharing) can borrow spectral resources from each other for short time frames (order of milliseconds). For each of these spectrum sharing scenarios, we can use either orthogonal or non-orthogonal spectrum sharing.

In orthogonal spectrum sharing, the operator that borrows the spectrum can use it exclusively. Hence, the operators will not cause interference to each others users. The drawback with orthogonal sharing is that it might not exploit all degrees of freedom or diversity in the wireless channels. In non-orthogonal spectrum sharing, two or more operators or neighboring cells of one operator, simultaneously use the same piece of spectrum at a given physical location. One drawback of such sharing is that the operators or base stations cause interference to each others' users. This can substantially degrade the performance of the mobile users. On the other hand, the flexibility increases and we can potentially increase the number of served users or the data rate of the users with non-orthogonal sharing.

In this thesis, we focus on the downlink of the non-orthogonal spectrum sharing scenario. We use the interference channel (IC) as a model to understand the impact of the interference and how the operations can be coordinated. 
An IC consists of $K$ transmitter (TX)-receiver (RX) pairs, e.g., base stationmobile user pairs, where each TX serves one RX. Since the TX-RX pairs operate simultaneously in the same frequency band, they cause interference to each other. To suppress the interference, we can employ multiple antennas at the TXs. Then, the TXs are able to steer, or beamform, the radiated power such that they provide the intended RXs with strong signals and cause weak interference to the unintended RXs. The IC with multiple-antennas TXs and single-antenna RXs constitutes a multiple-input single-output (MISO) IC.

In the first part of this thesis, we gain understanding of the fundamental performance limits of the two-user MISO IC, i.e., there are two TX-RX pairs. We study various achievable rate regions and methods for computing them. The first contribution is on efficient computation of the outer boundary of the rate region when the TXs have instantaneous channel state information (CSI) and the receivers are capable to perform successive interference cancellation. We split the problem in four subproblems corresponding to the different combinations of decoding strategies (decode interference or treat it as noise). The optimization problems we solve are scalar and quasi-concave and can be solved either in closed form or numerically by a gradient ascend method. The second contribution is on the ergodic rate region with statistical CSI. We characterize the transmit covariance matrices which potentially yield points on the outer boundary of the rate region. Using these characterizations, we can reduce the search space in the design of the optimal transmit covariance matrices. The third contribution considers a slow-fading channel and provides four different definitions of outage rate regions. These definitions depend on whether there is instantaneous or statistical CSI and whether outage is declared individually or in common. In the two latter contributions, the RXs treat interference as noise.

The second part of this thesis addresses the resource allocation problem in a small cellular network. The first contribution considers the inter-operator spectrum sharing problem in a single cell. The results illustrate that if user selection is not possible and there are always users to serve for both operators, there is no gain of non-orthogonal spectrum sharing over orthogonal sharing. For a similar setup, the second contribution considers the user selection problem. The base stations select one user each to serve. The computational complexity of optimal user selection is high. Therefore, we propose to use simple beamforming schemes in order to select a user pair. Once a pair is chosen, we use optimal beamforming. The performance loss of this algorithm, compared to using optimal beamforming vectors for the scheduling is negligible. 


\section{Populärvetenskaplig sammanfattning}

Under de senaste decennierna har vi kunnat bevittna en snabb utveckling och ständigt ökad efterfrågan av trådlös, eller mobil, kommunikation. Detta har lett till att det frekvensutrymme, eller spektrum, som lämpar sig för trådlös kommunikation håller på att ta slut. För att även fortsättningsvis kunna erbjuda trådlösa tele- och datakommunikationstjänster av hög kvalitet är det nödvändigt att det befintliga frekvensutrymmet utnyttjas effektivare än vad det görs idag.

En teknik som har framförts för att använda det tillgängliga spektrumet på ett mer effektivt sätt är så kallad spektrumdelning. Detta innebär att olika basstationer eller operatörer samsas om en bit av spektrumet på en given fysisk plats genom att låna det av varandra vid behov. På detta sätt får de utökad flexibilitet när det gäller att utnyttja spektrumet.

Spektrumdelning kan antingen ske ortogonalt eller ickeortogonalt. Med ortogonal spektrumdelning menas att en operatör kan låna en bit av det spektrum som tillhör en annan operatör. Den första operatören kan använda detta spektrum utan att den andra operatören är aktiv där. På så sätt kommer operatörerna inte att störa varandras användare. I fallet med ickeortogonal spektrumdelning använder båda operatörerna spektrumet samtidigt. På detta sätt skapas större flexibilitet vid optimeringen av användandet av spektrumet. Dock innebär ickeortogonal spektrumdelning att operatörernas basstationer och mobila användare kommer att störa varandra. Vi säger att de skapar interferens.

Denna avhandling fokuserar på ickeortogonal spektrumdelning och kommunikationen från basstationer till användare, d.v.s. den så kallade nedlänken. 
Genom att utrusta varje basstation med flera antenner har de möjlighet att använda så kallad lobformning för att styra den utsända effekten. På så sätt kan en basstation sända en stark signal till de mobila användare som den betjänar samtidigt som den undertrycker interferensen, eller störningen, som den orsakar de användare som den inte betjänar.

I den första delen av denna avhandling studeras vilka teoretiska datatakter som kan uppnås för en interferenskanal som består av två sändarmottagarpar när sändarna är utrustade med multipla antenner. I fallet med två eller flera sändar-mottagarpar är det meningsfullt att studera så kallade datataktsregioner. Dessa beskriver vilka datatakter som kan uppnås samtidigt för de två paren. Vi fokuserar på definitioner och effektiv beräkning av dessa regioner.

I den andra delen av avhandlingen studeras resursallokering i ett litet trådlöst nätverk. Här består nätverket av två basstationer som var och en betjänar flera mobila användare. Vi visar numeriskt att om användarval ej är möjligt så är vinsterna med ickeortogonal spektrumdelning över ortogonal spektrumdelning relativt liten. För fallet då användarval är möjligt föreslår vi en beräkningseffektiv metod för att välja användare i detta nätverk. Med användarval visar det sig vara fördelaktigt med ickeortogonal spektrumdelning. 


\section{Acknowledgments}

My deepest gratitude goes to my supervisor Prof. Erik G. Larsson. Thank you for giving me the opportunity to pursue my doctoral studies in this excellent research group. Without your guidance, supportm and patiance, I would never have been able to accomplish this work.

A special thank goes also to my co-supervisor Dr. Eleftherios Karipidis. I really learned a lot from you, especially when it comes to presenting the outcomes from the research, both in writing and orally.

I am thankful to all partners of the SAPHYRE project. The cooperation within the project gave me research ideas and it helped me to understand where my research belongs in a greater context. This and other cooperations I have participated in led to several common publications with many coauthors. Thank you all! Special thanks go to Prof. Eduard A. Jorswieck, Dr. Rami Mochaourab, and Andreas Gründinger for several interesting research discussions.

Many thanks go to all my colleagues in the Communication Systems division and the neighboring Information Coding Group. Especially, I would like to thank Dr. Mikael Olofsson, Prof. Robert Forchheimer, and Carina Lindström. Mikael for being my mentor in teaching related matters. I got many useful tips that helped me to develop as a teacher. Robert, who was my co-supervisor for two years in the beginning of my $\mathrm{PhD}$ studies. Carina, for being the coordinator we need and for always trying to spread a cheerful atmospher in the corridor.

Finally, I would like to thank my parents Ia Lindgren Lindblom and Bo Lindblom as well as my sister Kristina Lindblom and my brother Ludvig Lindblom with their families for their encouragement. 


\section{Abbrevations}

$\begin{array}{ll}\text { 3GPP } & \text { The 3rd Generation Partnership Project } \\ \text { BC } & \text { Broadcast Channel } \\ \text { BS } & \text { Base Station } \\ \text { cdf } & \text { cumulative distribution function } \\ \text { CoMP } & \text { Cooperative Multi-Point } \\ \text { CR } & \text { Cognitive Radio } \\ \text { CSI } & \text { Channel State Information } \\ \text { DPC } & \text { Dirty Paper-Coding } \\ \text { FFR } & \text { Fractional Frequency Reuse } \\ \text { GSM } & \text { Global System for Mobile Communications } \\ \text { HetNet } & \text { Heterogeneous Network } \\ \text { IC (or IFC) } & \text { Interference Channel } \\ \text { KKT } & \text { Karush-Kuhn-Tucker } \\ \text { LTE } & \text { Long Term Evolution } \\ \text { MAC } & \text { Multiple-Access Channel } \\ \text { MIMO } & \text { Multiple-Input Multiple-Output } \\ \text { MISO } & \text { Multiple-Input Single-Output } \\ \text { MR } & \text { Maximum Ratio } \\ \text { MRT } & \text { Maximum Ratio Transmission } \\ \text { MS } & \text { Mobile Station } \\ \text { MSLNR } & \text { Maximum Signal-to-Leakage-plus-Noise Ratio } \\ \text { NP } & \text { Non-deterministic Polynomial-time } \\ \text { pdf } & \text { probability density function } \\ \text { PO } & \text { Pareto Optimal } \\ \text { RX } & \text { Receiver } \\ \text { SDP } & \text { Semi-Definite Program } \\ \text { SIC } & \text { Successive Interference Cancellation } \\ \text { SIMO } & \text { Single-Input Multiple-Output } \\ \text { SINR } & \text { Signal-to-Interference-plus-Noise Ratio } \\ \text { SISO } & \text { Single-Input Single-Output } \\ & \end{array}$


SNR

SOCP

$\mathrm{SU}$

TX

UMTS

$\mathrm{ZF}$
Signal-to-Noise Ratio

Second-Order Cone Program

Single-User

Transmitter

Universal Mobile Telecommunication System

Zero-Forcing 


\section{Contents}

$\begin{array}{ll}\text { Abstract } & \mathbf{v}\end{array}$

Populärvetenskaplig sammanfattning (in Swedish) vii

$\begin{array}{ll}\text { Acknowledgments } & \text { ix }\end{array}$

Abbreviations $\quad x i$

I Introduction 1

1 Background: Why Sharing the Spectrum? 3

2 Techniques for Spectrum Sharing $\quad 7$

2.1 Inter-Operator Spectrum Sharing . . . . . . . . . . . . . 7

2.2 Dealing with Interference . . . . . . . . . . . . . . . . . 9

2.2.1 Fractional Frequency Reuse . . . . . . . . . . . 10

2.2.2 Multiple Antennas . . . . . . . . . . . . . . 12

2.3 Alternative Techniques for Improving Spectral Efficiency . . 13

2.3.1 Coordinated Multi-Point Transmission . . . . . . . 13

2.3.2 Cognitive Radio . . . . . . . . . . . . . . . . . . . . 14

2.3.3 Very Large Antenna Systems . . . . . . . . . . . . 15

2.3.4 Heterogeneous Networks . . . . . . . . . . . 15

2.4 Limitations on Cooperation . . . . . . . . . . . . . 16

3 Information Theoretical Modeling $\quad 19$

3.1 Notation . . . . . . . . . . . . . . . . . . . 20

3.2 Multiple-Access Channel . . . . . . . . . . . . . . . . . . 21

3.2.1 The Special Case of $K=1$. . . . . . . . . . . 23

3.2.2 The Special Case of Gaussian SIMO MAC . . . . . . 23

3.2.3 The Special Case of Two-User Gaussian SISO MAC 24 
3.3 Broadcast Channel . . . . . . . . . . . . . . . . . . . 25

3.3.1 The Special Case of Gaussian MISO BC . . . . . . 28

3.3.2 The Special Case of Two-User Gaussian SISO BC . $\quad 29$

3.4 Interference Channel . . . . . . . . . . . . . . . . . 29

4 Contributions of the Thesis and Open Research Directions 33

4.1 Achievable Rate Regions . . . . . . . . . . . . . . . . . 33

4.2 Resource Allocation and Spectrum Sharing . . . . . . . . 38

4.3 Included Publications . . . . . . . . . . . . . . . . 39

4.4 Publications not Included in the Thesis . . . . . . . . . 42

II Achievable Rate Regions 51

A Efficient Computation of Pareto Optimal Beamforming Vectors for the MISO Interference Channel with Successive Interference Cancellation 53

1 Introduction . . . . . . . . . . . . . . . 56

1.1 Contributions and Organization . . . . . . . . 58

$1.2 \quad$ Notation . . . . . . . . . . . . . . . . . . . . . . . . 59

2 System Model . . . . . . . . . . . . . . . . . . . 60

3 Achievable Rate Region of SIC Capable RXs . . . . . . . 61

4 Both RXs Treat the Interference as Noise . . . . . . . . . 64

$4.1 \quad$ Numerical Method . . . . . . . . . . . . . . . . 65

4.2 Closed-Form Parameterization _. . . . . . . . . 69

5 Only one RX Decodes the Interference . . . . . . . . . . 74

6 Both RXs Decode the Interference . . . . . . . . . . . 78

7 Numerical Illustrations . . . . . . . . . . . . . . . . . . . . 84

$7.1 \quad$ Computational Complexity . . . . . . . . . . . . 88

8 Conclusion . . . . . . . . . . . . . . . . . . . 89

B Parameterization of the MISO IFC Rate Region: The Case of Partial Channel State Information $\quad 95$

1 Introduction . . . . . . . . . . . . . . . . 98

2 System Model . . . . . . . . . . . . . . . . . . . . . . . . 99

3 The Achievable Rate Region . . . . . . . . . . . . . . . . 100

4 Necessary Conditions for the Pareto Boundary . . . . . . . 101

$5 \quad$ Special Cases . . . . . . . . . . . . . . . . . . . 106

6 Numerical Results . . . . . . . . . . . . . . . . . . . . 106

7 Conclusions . . . . . . . . . . . . . . . . . . . 107

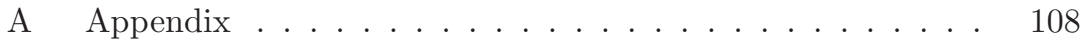


C Selfishness and Altruism on the MISO Interference Channel: The Case of Partial Transmitter CSI 113

1 Introduction . . . . . . . . . . . . . . . . . 116

2 System Model . . . . . . . . . . . . . . . . . . 117

3 Closed-Form Ergodic Rate Expression . . . . . . . . . . . . 117

4 Nash-equilibrium Strategy . . . . . . . . . . . . . . . . 118

$5 \quad$ Zero-forcing Strategy . . . . . . . . . . . . . . . . . . . 119

6 Pareto-Optimal Strategies . . . . . . . . . . . . . . 120

$7 \quad$ Numerical Example . . . . . . . . . . . . . . . . . 122

8 Discussion . . . . . . . . . . . . . . . . . . . 123

D Achievable Outage Rate Regions for the MISO Interference Channel

1 Introduction . . . . . . . . . . . . . . . . . . . 130

2 System Model . . . . . . . . . . . . . . . . . . . 131

3 Outage Rate Region for Instantaneous CSI . . . . . . . . . 131

3.1 Common Outage Rate Region for Instantaneous CSI 132

3.2 Individual Outage Rate Region for Instantaneous CSI 132

4 Outage Rate Regions for Statistical CSI . . . . . . . . . . . 137

4.1 Common Outage Rate Region for Statistical CSI . . 137

4.2 Individual Outage Rate Region for Statistical CSI . 137

4.3 Outage Probabilities for Statistical CSI . . . . . . 138

5 Numerical Example. . . . . . . . . . . . . . . . . . . . . 140

6 Comparison of Regions . . . . . . . . . . . . . . . . . . . . 142

7 Conclusions .................... 145

III Resource Allocation and Spectrum Sharing 149

E Does Non-Orthogonal Spectrum Sharing in the Same Cell Improve the Sum-Rate of Wireless Operators? 151

1 Introduction . . . . . . . . . . . . . . . . . . . . . . 154

2 Models . . . . . . . . . . . . . . . . . 155

3 Non-Orthogonal Sharing: The MISO IC . . . . . . . . . . . 157

3.1 Lower (Achievable) Bounds Using Linear Beamforming 158

3.2 Upper Bounds . . . . . . . . . . . . . . . . . . . . . 159

4 No Sharing: The MISO BC . . . . . . . . . . . . . 160

4.1 Lower (Achievable) Bounds Using Linear Beamforming 161

4.2 Upper Bounds . . . . . . . . . . . . . . . . . . . 162

5 Numerical Comparisons ................ 163 
6 Conclusions .................... 165

F Joint User Selection and Beamforming Schemes for InterOperator Spectrum Sharing $\quad 169$

1 Introduction . . . . . . . . . . . . . . . . . . . 172

2 System Model . . . . . . . . . . . . . . . . . . . . 173

3 Scheduling Algorithms . . . . . . . . . . . . . . 175

4 Simulation Results . . . . . . . . . . . . . . . . . . . 177

5 Concluding Remarks . . . . . . . . . . . . . . 179 


\section{Part I}

\section{Introduction}





\section{Chapter 1}

\section{Background: Why Sharing the Spectrum?}

The demand for mobile data traffic is steadily increasing. The number of mobile devices as well as the amount of data traffic per device increases. In a report from early 2013, [1], Cisco forecasts that, by the end of 2013, the number of mobile devices will exceed the number of people on the earth and between 2012 and 2017 there will be a 13-fold increase of mobile data traffic. On the other hand, the spectrum suitable for mobile broadband is quite limited by hardware constraints and channel behavior [2]. At frequencies below $300 \mathrm{MHz}$, the antennas cannot be made small enough to fit into mobile devices and at frequencies higher than $5 \mathrm{GHz}$, the radio signal is difficult to penetrate buildings and the antenna gain must be high to maintain the signal-to-noise ratio (SNR). Moreover, regulations have led to a situation where the spectrum is allocated to very specific purposes. The combination of increasing demand for mobile data traffic and limited spectral resources requires a more efficient use of the spectral resources.

Traditionally, the spectral resources for wireless cellular systems have been divided among the operators in spectrum auctions. In these auctions, the spectrum is split into chunks of bandwidth which are exclusively licensed to the operators which win the auction and the spectrum is licensed for a long time (typically a decade or longer). Not only is the spectrum divided between the operators, it is also divided among different technologies, e.g., Global System for Mobile Communications (GSM), Universal Mobile Telecommunications System (UMTS), and Long Term Evolution (LTE), within each 
operator. Moreover, the frequency planning was performed such that neighboring cells do not use the same frequency in order to avoid creating inter-cell interference. However, such planning comes with the cost of low spectral efficiency since only a fraction of the total spectrum is used in each geographical area. Also, such allocation of spectrum to operators, technologies, and cells does not offer much of flexibility in the use of the spectrum.

Spectrum sharing has been proposed as a technique for more efficient usage of the spectrum [3]. The idea is that technologies, cells, and even operators share the spectrum. For example, if operator A has no available spectral resources while operator B has, a mobile user of operator A can be moved to operator B for a short period of time (order of milliseconds or seconds). Another solution might be that operator A borrows a piece of spectrum from operator B. By allowing this kind of sharing, the spectrum can be better utilized and the operators can serve more mobile users. Spectrum sharing can be characterized and categorized in two features, namely cooperation or coexistence and sharing among equals or primary-secondary sharing [4]. In a spectrum sharing scenario based on cooperation, devices belonging to different operators, standards and cells must cooperate with each other to avoid causing too much of mutual interference. This requires an infrastructure for communication between the devices. This infrastructure that consists of, e.g., backbone network and communication protocols, must be supported by the devices in the actual frequency band. In a coexistence model, devices avoid interference without using explicit signaling. This can be accomplished by spectrum sensing where the devices identify the pieces of the spectrum where the interference is low. In the primary-secondary sharing, some systems act as primary users and dictate to the secondary systems that they are not allowed to cause harmful interference to the primary system. In the sharing among equals case, the devices have equal rights to use the spectrum which typically is more flexible than the primary-secondary sharing. On the other hand, in the equal priority sharing case, all devices must have the incentive to limit the mutual interference. In total we have four combinations of the features, where this thesis focuses on cooperation for sharing among equals.

For the most flexible way of sharing the spectrum, a piece of spectrum is used simultaneously by two, or more, technologies, cells, or operators. This is the so-called non-orthogonal spectrum sharing. In such scenario the different units of the system will cause interference to each other and we have a so-called interference network. In the example illustrated in Fig. 1.1, we have two base stations operating in the downlink, i.e., a base station sends 


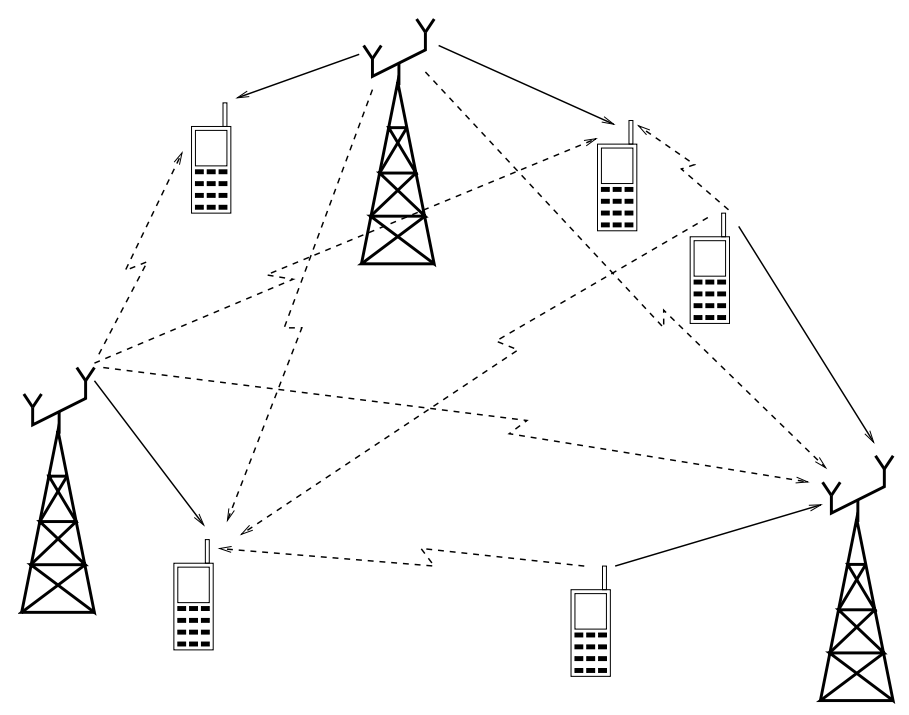

Figure 1.1: Illustration of wireless communication in a non-orthogonal spectrum sharing scenario. Solid lines correspond to intended signals whereas dashed lines are unwanted interference.

data to many mobile users, and one base station operating in the uplink, i.e., many mobile users are sending data to the base station. The base station working in the downlink will not only send data to its intended users but it will cause interference to the other base station receiving data in the uplink as well. On the other hand the mobile users of the second cell (uplink communication) cause interference to the mobile users of the first cell (downlink communication). If these interference sources are not coordinated, the system degradation can be substantial.

Another, but related, technique for increasing coverage and spectral efficiency while reducing the total hardware cost is infrastructure sharing. For example, two operators can share the backbone network, the mast, the base station, or even the antennas. Infrastructure sharing has already been implemented, for example in Sweden, where operators are allowed to share the network as long as each operator with its own equipment covers at least $30 \%$ of the population. The introduction of UMTS in Sweden led to a situation where four operators together, in pairs, formed two network companies. The main driver for forming such coalitions, was the requirement from the regulatory agency that an operator should cover $70 \%$ of the population [5]. In this thesis, we do not consider the infrastructure sharing problem. 
Parts of this work have been performed within the SAPHYRE (Sharing physical resources - mechanisms and implementations of for wireless networks) project which was funded by the European Union within framework program seven. The visions of SAPHYRE were to show how voluntary sharing of physical and infrastructure resources enables a fundamental gain in the efficiency of spectrum utilization, develop the enabling technology that facilitates such voluntary sharing, and determine determine the key features of a regulatory framework that supports such voluntary sharing. Variations of spectrum sharing are now becoming accepted by the industry as tool for future spectrum management, e.g., see the recently published white paper [6].

Though it is not a part of this thesis, regulatory and business aspects of spectrum sharing are important for the success of inter-operator spectrum sharing. The SAPHYRE project [7] points out three different types of spectrum sharing. In intra-operator spectrum sharing, an operator shares its spectral resources between different access technologies. In cooperative sharing, two or more operators share the spectrum that was licensed to them in the traditional way. In the spot-market scenario, the regulatory body does not license spectrum in the traditional way. Instead it allows the operators to share the spectrum and then charge them based on the used amount of spectrum. Appropriate pricing mechanisms are as important for enabling spectrum sharing as the actual techniques. The pricing mechanism should be beneficial for the operators that share spectrum and it should stimulate such initiative. It is important that the operators maximize their revenue from their own spectrum while it is in line with the regulations. One regulatory issue is how monopoly situations are avoided. As the cooperation between operators increases, the operators tend to act as a single operator [8].

In the following chapters, we will describe some techniques proposed to overcome the spectrum shortage by using the spectrum in a more flexible manner. In Ch. 2, we start by describing techniques for intra-operator sharing, i.e., frequency planning within each operator. Then, we describe the so-called inter-operator spectrum sharing. The techniques enabling intra-operator spectrum sharing are also enablers for inter-operator spectrum sharing. We will briefly discuss some alternative techniques for spectrum sharing such as coordinated multi-point (CoMP), cognitive radio (CR) and heterogeneous networks (HetNets). In Ch. 3, we identify several information theoretical models which occur when the spectrum is shared. For each of the models, we summarize the key results. Ch. 3 also discusses the fundamental limits of cooperation among base stations and operators. In Ch. 4, we present the contributions of the thesis and put it in a greater context. 


\section{Chapter 2}

\section{Techniques for Spectrum Sharing}

In this chapter, we discuss the different techniques for inter- and intraoperator spectrum sharing in this thesis. In Sec. 2.1, we explain what we mean by spectrum sharing. We mainly focus on inter-operator spectrum sharing and consider both orthogonal and non-orthogonal sharing. We will see that non-orthogonal spectrum sharing, which is the focus of this thesis, incurs inter-cell and inter-operator interference which can substantially limit, e.g., the sum-rate of the system. Therefore, in Sec. 2.2, we discuss techniques for mitigating interference. Finally, since spectrum sharing is a general term for a large number of techniques, we present some alternative techniques for increasing the spectral efficiency in wireless networks in Sec. 2.3. In Sec. 2.4, we briefly discuss some fundamental limitations of cooperation in interference networks.

\subsection{Inter-Operator Spectrum Sharing}

In this section we describe techniques for spectrum sharing. This exposition and the terminology follow that of [9]. Traditionally, a fixed allocation of the spectrum has been performed. The operators got the rights to exclusively use a certain amount of spectrum. Within the spectrum chunk of each operator, different technologies such as GSM, UMTS, and LTE are allocated to fixed subbands. This is illustrated in Fig. 2.1 (a). This does not provide much of flexibility in the allocation of the mobile users. If we allow an operator to 

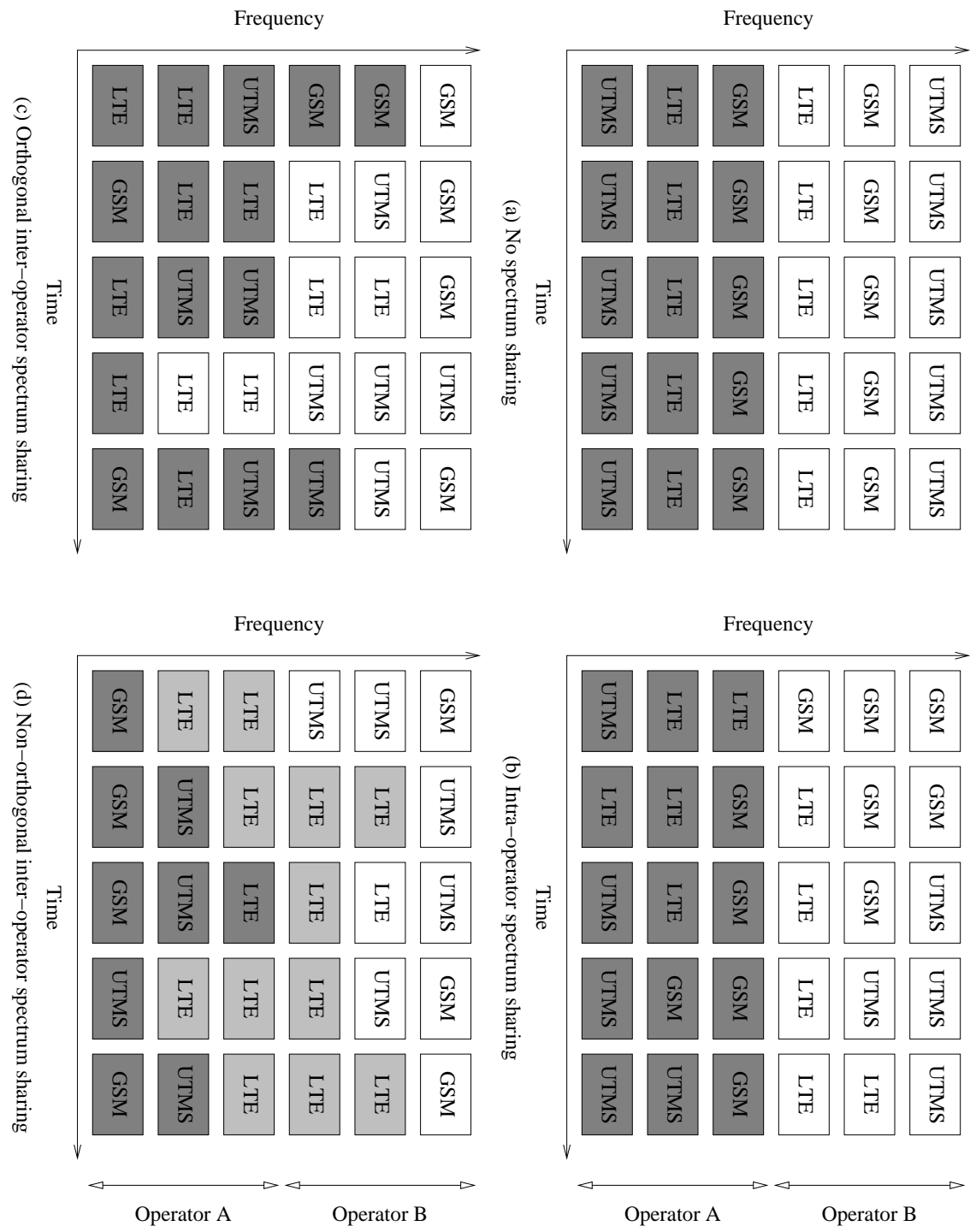

Figure 2.1: Illustration of spectrum sharing scenarios, freely reproduced from [7]. White and dark gray time/frequency resources are used exclusively by operators A and B, respectively. Light gray time/frequency resources are non-orthogonally shared between the two operators. 
allocate its spectrum chunk based on the current demand, as illustrated in Fig. 2.1 (b), we increase the flexibility since users and technologies can be moved in frequency. To further increase the flexibility in the networks, we can let one operator temporarily borrow some resource from the other operator if it has resources available. In the orthogonal inter-operator spectrum sharing scenario illustrated in Fig. 2.1 (c), an operator can use the shared subband exclusively. That is, there will not be any inter-operator interference. Finally, in the non-orthogonal scenario both operators use the shared spectrum simultaneously. Hence, the operators will cause inter-operator interference to each others' users. It should be noted that the operators might not share their entire spectrum, but keep some of it for their exclusive use. How the spectrum is divided between private and shared chunks is a question for business and regulatory authorities.

If the two operators own one cellular network each, the non-orthogonal interoperator spectrum sharing leads to a situation of two overlapping networks as illustrated in Fig. 2.2. In the worst interference scenario, all cells of both operators are active. If this interference is not appropriately coordinated, it will substantially degrade the performance of the networks, which is more quantitatively illustrated in Paper F.

\subsection{Dealing with Interference}

When considering non-orthogonal spectrum sharing, there will be several sources of interference as illustrated in Fig. 1.1. In the most general forms there will be intra- and inter-cell interference as well as inter-operator interference. Intra-cell interference is caused when a base station serves multiple users in the same time-frequency slot. Inter-cell interference is caused by the base station or the users of one cell to the base station or users of a neighboring cell when the two cells are active in the same time-frequency slot. Especially, the performance of cell-edge users can be severely degraded due to inter-cell interference. Finally, two operators that are active in the same time-frequency slot cause inter-operator interference to each other. Due to the overlapping network, all users will be affected by the inter-operator interference. All these interference sources can substantially degrade the performance of the wireless network. Here, we discuss two techniques that deal with this interference. 


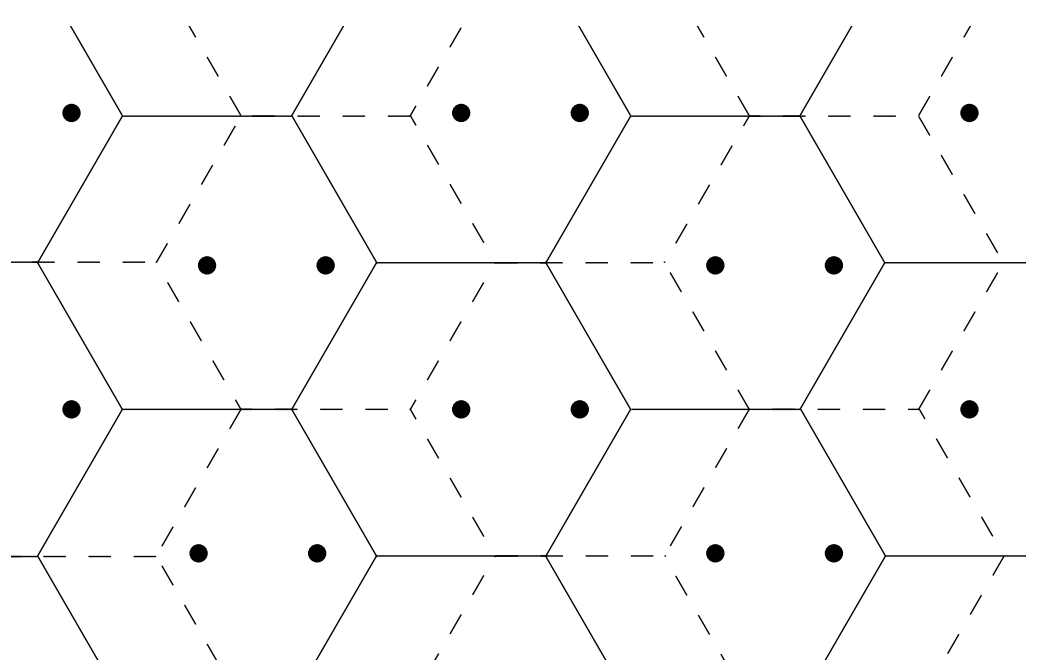

Figure 2.2: Illustration of the two overlapping cellular networks in a nonorthogonal spectrum sharing scenario. We can assume that the cells with solid and dashed borders belong to operators A and B, respectively. The black dots represent the base stations that are placed in the cell center.

\subsubsection{Fractional Frequency Reuse}

The classical way of avoiding inter-cell interference has been to divide the entire available frequency band into several orthogonal, but equally large, subbands. These subbands are then allocated to the different cells such that two neighboring cells do not use the same subband. This is illustrated in Fig. 2.3, where we have three different subbands. Since there is always one cell between two cells that use the same subband, the inter-cell interference will be made small thanks to the path-loss. On the other hand, for the example in Fig. 2.3, each cell can only use one third of the entire spectrum and, hence, the spectral efficiency is low.

Fractional frequency reuse (FFR) has been proposed as a solution that improves the spectral efficiency while it mitigates the inter-cell interference [10]. The strict FFR splits the spectrum into an inner and an outer part [11]. The inner part is the same subband for all cells and it is allocated to the users close to the base station. Therefore, the base station can use low power to 


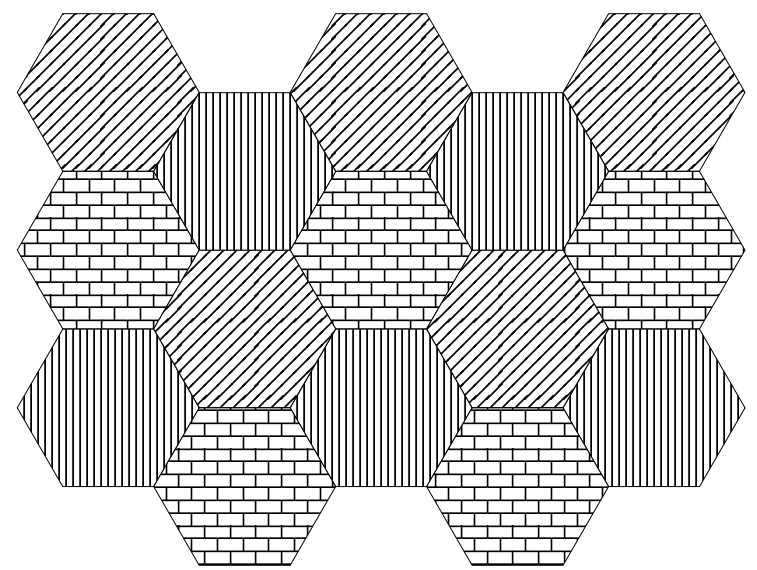

Figure 2.3: Illustration of frequency reuse-3. The different patterns relate to the disjoint set of frequencies.
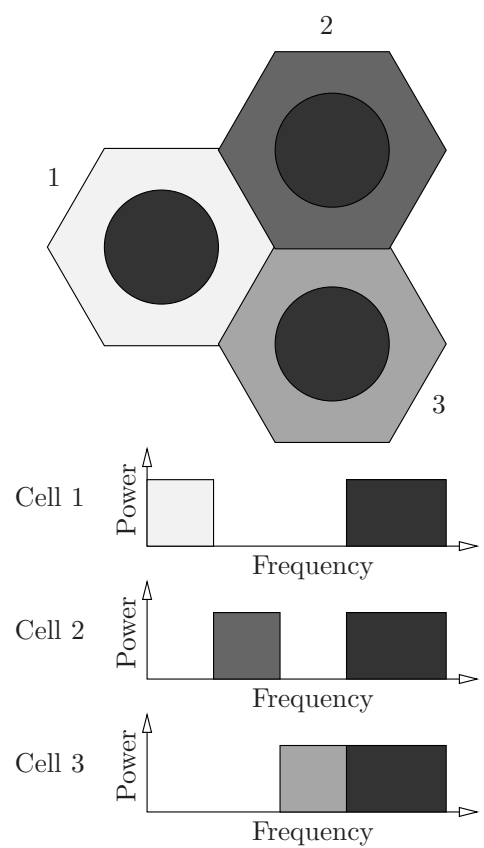

(a) Strict FFR
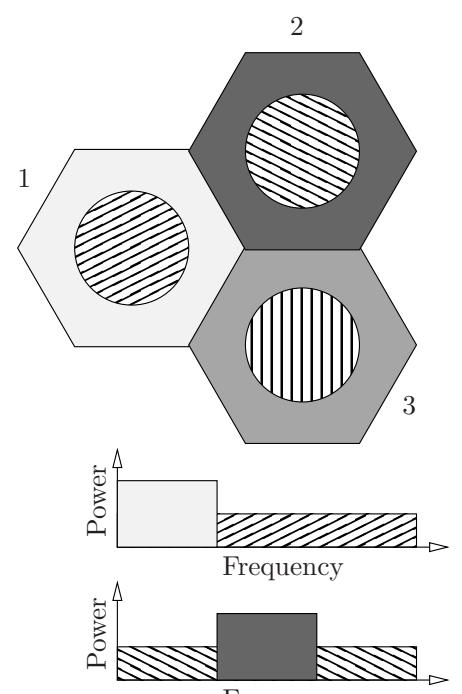

Frequency

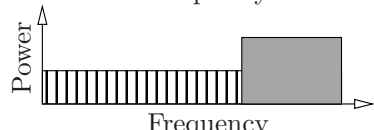

(b) Soft FFR

Figure 2.4: Illustration of fractional frequency reuse. 
serve these users. Fractions of the outer part are allocated to the cell-edge users with a frequency reuse factor larger than one, e.g., as in Fig. 2.3 where the reuse factor is three. The implementation of strict frequency reuse is quite simple but it suffers from reduced spectral efficiency since each cell cannot use the entire spectrum. The strict FFR is illustrated in Fig. 2.4 (a).

Soft FFR is an alternative solution that has full spectral efficiency since each cell can use the entire bandwidth. However, there will be power limitations on some subbands [12]. The spectrum is again divided according to some frequency reuse factor larger than one, e.g., as in Fig. 2.3. This frequency allocation is used for the cell-edge users. However, all other subbands are used for the users close to the base station as illustrated in Fig. 2.4 (b). If the transmit power intended for the users in the inner part is small, the interference towards the neighboring cells will not be too harmful.

\subsubsection{Multiple Antennas}

By employing multiple antennas as transmitters and receivers, a number of potential gains can be achieved [13]. In this exposition, we consider a link with $n_{T}$ transmit antennas and $n_{R}$ receive antennas, then a so-called multiple-input single-output (MIMO) system. The different transmit and receive antennas can potentially belong to several different units.

Array gain is achieved by coherent combining effects at transmitters and receivers. In that way the average received SNR can increase. The array gain depends on the number of available antennas and it requires accurate channel knowledge at both receivers and transmitters. Diversity gains are achieved by transmitting over multiple fading paths in time, frequency, or space. Spatial diversity is preferable since it does not incur bandwidth costs. Diversity gains can be achieved even if the transmitters lack channel knowledge. Spatial multiplexing gains provides a capacity increase that is linear in $\min \left\{n_{T}, n_{R}\right\}$ if the scattering is rich enough. We say that the system provides $\min \left\{n_{T}, n_{R}\right\}$ degrees-of-freedom (DoF). This gain is achieved by transmitting multiple data streams from the coded across the different antennas and separating the streams at the receiver side. In a network of multiple mobile users, we have multi-user diversity gain which arises from the fact that with high probability there are users with good channels. Finally, and most importantly for the problems studied in this thesis, is that multiple antenna systems can provide interference reduction. The idea is 
that the transmitter side can use beamforming to steer power in such way that the interference towards unintended receivers is avoided. At the receiver side, the multiple antennas can be used to filter out unintended signals. We can compare this with the human ears which can be turned in the direction the intended sound comes from.

It should be noted that all these gains cannot be achieved simultaneously they are conflicting objectives. With a few antennas we can maybe provide some spatial multiplexing, but not as much as $\min \left\{n_{T}, n_{R}\right\}$, and some interference reduction. Then there might not be any spatial dimensions left to provide array gains. Hence, there is a fundamental trade-off in the use of multiple antennas. For a multi-user network with multi-antenna transmitters and receivers, there is also the trade-off between providing full spatial multiplexing to a few users, i.e., sending many data streams to them, or providing many users with a few data streams each [14]. In the latter case, receiver dimensions which are not used for signal reception can be used for reducing the experienced interference.

\subsection{Alternative Techniques for Improving Spectral Efficiency}

Inter-operator spectrum sharing is one of many approaches for better utilization of the wireless spectrum. In this section, we briefly describe a few other techniques for increasing the spectral efficiency in wireless networks. Some of these techniques can very well be used in an inter-operator spectrum sharing scenario. All of them are enablers for wireless network entities to share the spectrum.

\subsubsection{Coordinated Multi-Point Transmission}

Coordinated multi-point (CoMP), sometimes called cooperative multi-point, transmission is a technique where several base stations cooperate in order to avoid causing serious interference to mobile users and increase spectral efficiency. CoMP is limited to a single operator and it requires the base station to exchange channel state information (CSI) and, in some cases, user data via a high-data rate backbone network. CoMP uses frequency reuse one, which is similar to the non-orthogonal spectrum sharing scenario. 
Studies in the 3rd Generation Partnership Project (3GPP) categorized three different types of CoMP techniques. The first one is coordinated scheduling and coordinated beamforming. The base stations share CSI for multiple mobile users but the data intended for a user is available only at one base station. Actually this is the scenario we consider for inter-operator spectrum sharing. The second category is joint transmission, where the base stations share both CSI and user data. In such way, the set of base stations can virtually act as a single base station. The third category is base station selection where a mobile user is served by a single base station but the data for that user is available to all base stations. For more details about CoMP, e.g., see [15].

\subsubsection{Cognitive Radio}

Another solution that has been proposed in order to solve the scarcity of spectrum is cognitive radio (CR), see [16]. In this setup, there is a primary system that owns the spectrum and a secondary, potentially unlicensed, CR system that uses the spectrum at chance. The idea is that the CR system adapts to the wireless environment. Software-defined radio is one enabler of CR since it allows for being programmed and configured dynamically.

There are three major CR network paradigms [17]. In the underlay paradigm, the secondary system is aware of the interference it causes to the primary transmitters and receivers. The secondary system is allowed to use the licensed spectrum if it does not cause too much interference to the primary system. In the overlay paradigm, the secondary system knows the codebooks and messages of the primary system. This knowledge can be used to cancel or mitigate interference at primary and secondary receivers. The interweave paradigm is based on the idea of opportunistic communication. The secondary system utilizes that the spectrum, even though it is occupied, is underutilized most of the time [18] and transmits in the so-called spectrum holes. Knowledge about these holes can be acquired via spectrum sensing.

The CR can be seen as a enabler for inter-operator spectrum sharing. In a broader sense, inter-operator spectrum sharing benefits from cognitive and flexible transceivers. 


\subsubsection{Very Large Antenna Systems}

Very Large Antenna Systems, also called massive MIMO, has been proposed as a technique to accommodate more mobile users [19]. In the massive MIMO regime, we have tens of users and hundreds of base station antennas. In such a system, it is possible to form very narrow beams, making the interference to unintended users asymptotically vanishing when the number of transmit antennas approaches infinity. Also, under idealistic channel knowledge and hardware, the radiated transmit power is inversely proportional to the number of transmit antennas. Even for the scenario of uncoordinated base stations and rudimentary channel estimation, one can see impressive user data rate performance [19].

There are also several challenges associated with very large antenna systems [20]. These systems rely on accurate CSI. One of the most critical issues is that of pilot contamination in a multi-cell setup. Due to the contamination, the inter-cell interference does not vanish asymptotically as the number of base station antennas goes to infinity. Other issues that affect the accuracy of the CSI are hardware impairments. Despite the challenges, there seems to be a large potential in very large antenna systems.

As far as we know, very large antennas systems have not yet been studied in the context of inter-operator spectrum sharing. At least it is one potential solution to spectrum shortage problem that we try to solve by inter-operator spectrum sharing.

\subsubsection{Heterogeneous Networks}

Heterogeneous networks, also called multi-tier networks, have been proposed as a way to densify the wireless network and increase its spectral efficiency, e.g., see [21]. Basically, a traditional cell, the so-called macrocell, is splitted into smaller cells. As the cell sizes decrease the frequency reuse distance can decrease and, hence, the spectral efficiency increases. Also, the transmitted power can be decreased since the users come closer to the base stations. In a heterogeneous network, there are several levels, or tiers, of cells. A macrocell covers a rural area with a radius up to a few tens of kilometers. The macrocell base stations are generally mounted on ground-based masts and rooftops. A microcell base station is used to cover an outdoor area of radius less than two kilometers and is placed in urban areas with high mobile 


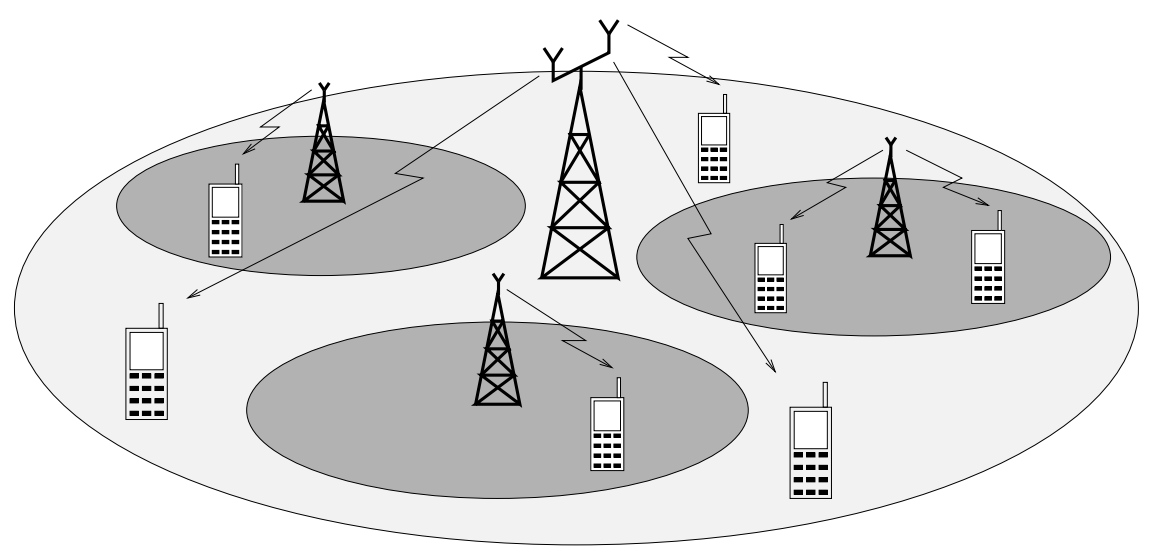

Figure 2.5: Illustration of a two-tier heterogeneous network. The large circle is a macrocell whereas the smaller, darker cells are microcells.

traffic density. The picocell is suitable for indoor use and covers distance up to a few hundreds of meters. A femtocell has a range of ten meters and is suitable for usage in homes.

In networks where small cells are used, a mobile user might switch cells more often than in a traditional network. This might lead to a scenario where a user might leave a cell before the previous hand-over is completed. To avoid this, high-speed users are assigned to the larger cells, whereas lowspeed users are assigned to the smaller cells. A small example of a two-tier network is illustrated in Fig. 2.5.

\subsection{Limitations on Cooperation}

There are fundamental limitations on, e.g., sum-rate performance in interference networks such as the one in Fig. 1.1. An upper bound on performance can be achieved by letting all transmitting units act as a single transmitter, i.e., they cooperate in beamforming and coding, and all receiving units act as a single receiver, i.e., we have a point-to-point MIMO link. The capacity of such a system increases unbounded with increasing SNR and the number of DoF is the minimum of the number total number of transmitting antennas 
and the total number of receiving antennas. The assumption that all transmitters and all receivers are cooperating is unrealistic for large networks. In the downlink, that would mean that all mobile users somehow have to be interconnected and all the base stations in the network have to share user data and CSI.

It is more realistic to assume that a few neighboring cells form a cooperation cluster and treat the out-of-cluster interference as noise. However, even if all transmitters and all receivers in the cluster are cooperating, the sum-rate will saturate at high SNR instead of growing unbounded [22]. The reason is that the out-of-cluster interference prevents the cooperating cluster from achieving the DoF we have in the point-to-point link. Another problem is the acquisition of CSI, where we must spend channel resources on the estimation of the channels. The estimates are affected by noise and the more of the signaling space we use for pilots, the better are the estimates. Also, as the cooperation cluster increases, more channels must be estimated and more of the signaling space must be used for this. On the other hand, the estimation phase competes with the payload data for signaling space. Hence there is a trade-off between spending resources on estimation or sending payload data. Even though there are drawbacks with cooperation, it might still provide significant performance gains as we illustrate in Paper F of this thesis. 


\section{Chapter 3}

\section{Information Theoretical Modeling}

To evaluate the potential gains of spectrum sharing and to devise algorithms for it, we want to be able to compute upper and achievable (lower) bounds on the capacity of the system. Unfortunately, the capacity of a system such as that in Fig. 1.1 is unknown. Achievable bounds under the assumption of Gaussian signaling and treating interference as noise can be computed using e.g., monotonic optimization [23], but it is an NP-hard problem even for the single-antenna case [24]. Still, we can gain some understanding by studying the three subsystems identified in Fig. 3.1. These are important information theoretical models, for which we will present some historical development and give the most important results. We consider the following models:

- Many transmitters send independent data to a single receiver. Usually, this model arises in the uplink, where many mobile devices communicate concurrently with a single base station. This is the so-called multiple-access channel (MAC).

- A single transmitter sends data to many receivers. This model arises in the downlink where a single base station concurrently transmits data to several mobile devices. This is the so-called broadcast channel (BC).

- Several transmitter-receiver pairs operate simultaneously in the same frequency band and, therefore, the pairs cause interference to each other. This model arises, e.g., at the cell edge where the mobile devices 


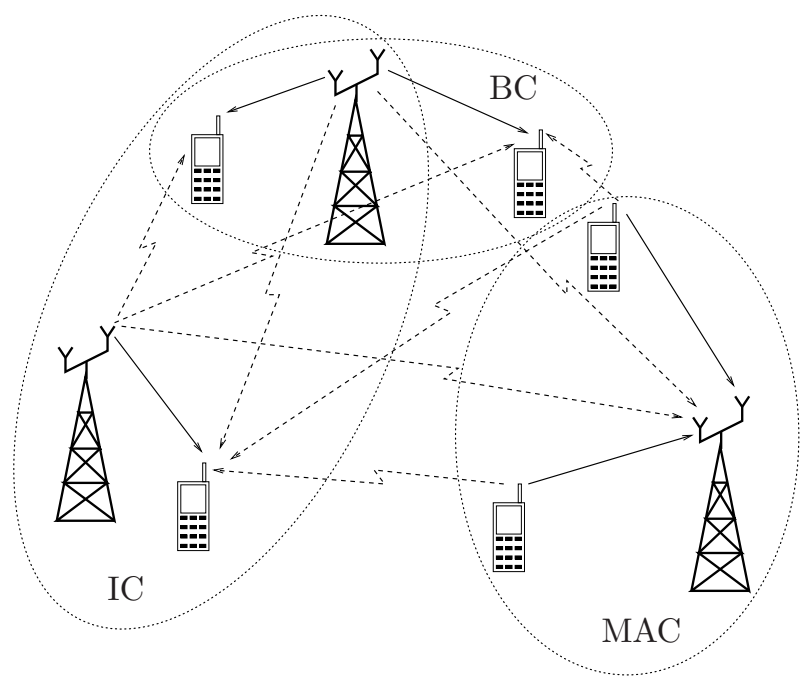

Figure 3.1: Identification of the MAC, BC, and IC in the general system model.

are close to each other. When one of the base stations transmits data to its intended mobile user, the mobile on the other side of the cell border will be interfered. This is an interference channel (IC).

In the exposition in Secs. 3.2-3.4, we assume that both the transmitters and receivers have instantaneous CSI. Moreover, we restrict this exposition to Gaussian channels.

\subsection{Notation}

Boldface lowercase and uppercase letters, e.g., $\boldsymbol{x}$ and $\boldsymbol{X}$, denote column vectors and matrices, respectively. The vectors $\mathbf{0}$ and $\mathbf{1}$ are the all-zeros and all-ones vectors, respectively. The former can also denote the all-zeros square matrix. $\boldsymbol{I}$ denotes the identity matrix. $\boldsymbol{y} \geq \boldsymbol{x}$ means that each component of $\boldsymbol{y}$ is greater than or equal to the corresponding element of $\boldsymbol{x}$. If $\boldsymbol{X} \succeq \mathbf{0}$, then we say that $\boldsymbol{X}$ is positive semi-definite. $\{\cdot\}^{T}$ and $\{\cdot\}^{H}$ denote the transpose and Hermitian (complex conjugate) transpose, respectively. The Euclidean norm of a vector $\boldsymbol{x}$ is denoted $\|\boldsymbol{x}\| \cdot \operatorname{diag}\{\boldsymbol{x}\}$ is the diagonal matrix 
constructed from the elements of the vector $\boldsymbol{x} \cdot \operatorname{tr}\{\cdot\}$ is the trace of a square matrix. By $|\cdot|$ we denote the determinant of a square matrix or the absolute value of a scalar. The operator $\mathrm{E}\{\cdot\}$ is the expectation of a random variable. By $\boldsymbol{x} \sim \mathcal{C N}(\mathbf{0}, \Psi)$ we say that $\boldsymbol{x}$ is a zero-mean complex-symmetric Gaussian random vector with covariance matrix $\Psi$.

\subsection{Multiple-Access Channel}

The MAC is a setup where $K \geq 2$ transmitters communicate simultaneously and in the same frequency band with a single receiver. The signals from the transmitters add up at the receiver, which also experiences additive thermal noise and, potentially, some uncoordinated interference. We assume that the messages of the different transmitters are independent. That is, the transmitters are not able to cooperate in the encoding of their messages.

Among the three models presented above, the MAC is the one that has the longest history and it is the only one for which the capacity region is completely understood for all possible channels. The single-input singleoutput (SISO) MAC, i.e., single-antenna transmitters and receiver, was first defined in [25]. The capacity region was established, in different ways, by [26-28]. The capacity region of the Gaussian SISO MAC, i.e., when the receiver experiences additive Gaussian noise was given in [29] and [30]. The capacity region of MIMO Gaussian MAC, i.e., the MAC with multi-antenna transmitters and receivers, was established in [31].

Here, we consider the most general form of the Gaussian MAC, that is the $K$-user Gaussian MIMO MAC as illustrated in Fig. 3.2. We give the capacity region and outline a scheme for computing the sum-capacity. The capacity regions for the Gaussian multiple-input single-ouput (MISO) (multiantenna transmitters and single-antenna receiver), single-input multipleoutput (SIMO) (single-antenna transmitters and multi-antenna receiver), and SISO MAC can be obtained as special cases of the Gaussian MIMO $\mathrm{MAC}$ region. Especially, we will present the capacity region of the two-user Gaussian SISO MAC.

We assume that the receiver is equipped with $n_{r}$ antennas and that transmitter $k$ is equipped with $n_{k}$ antennas. We let $\boldsymbol{H}_{k} \in \mathbb{C}^{n_{r} \times n_{k}}$ denote the channel matrix between the $k$ th transmitter and the receiver. By $\boldsymbol{e} \sim \mathcal{C N}(\mathbf{0}, \boldsymbol{I})$, we denote the additive Gaussian noise vector. Transmitter $k$ sends a symbol 


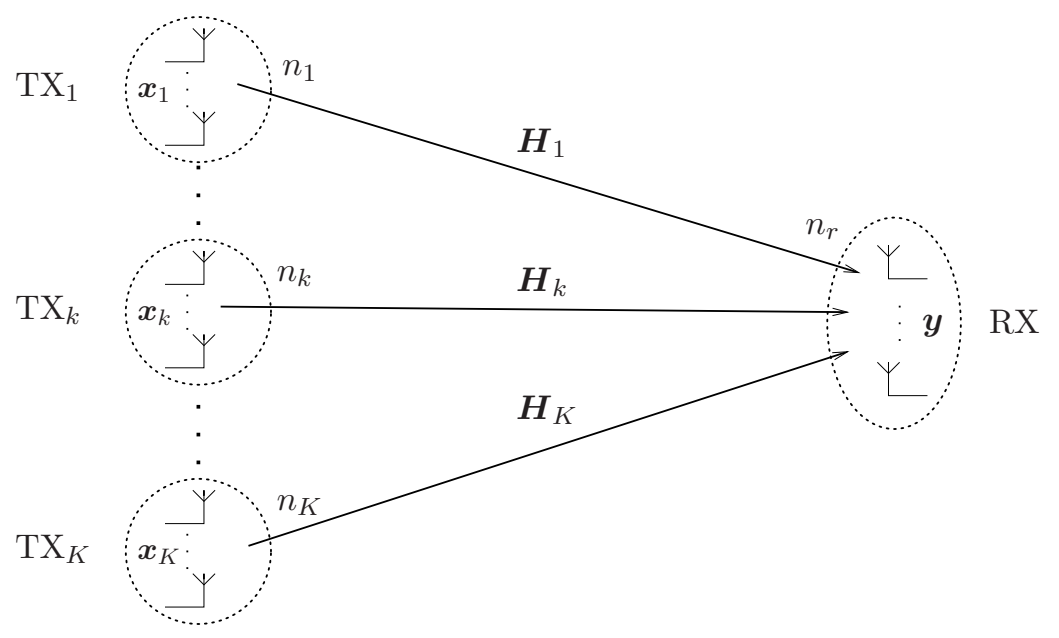

Figure 3.2: Illustration of a MIMO MAC.

vector $\boldsymbol{x}_{k}$ with covariance matrix $\boldsymbol{\Psi}_{k}$ which is subject to the normalized power constraint $\mathrm{E}\left\{\left\|\boldsymbol{x}_{k}\right\|^{2}\right\}=\mathrm{E}\left\{\operatorname{tr}\left\{\boldsymbol{x}_{k} \boldsymbol{x}_{k}^{H}\right\}\right\}=\operatorname{tr}\left\{\boldsymbol{\Psi}_{k}\right\} \leq 1$. Therefore, the matched-filtered, symbol-sampled complex baseband signal at the receiver is

$$
\boldsymbol{y}=\sum_{k=1}^{K} \boldsymbol{H}_{k} \boldsymbol{x}_{k}+\boldsymbol{e}
$$

The following theorem [31] gives the capacity region of the $K$-user Gaussian MIMO MAC.

Theorem 1. The capacity region of the Gaussian MIMO MAC is the set of rate points $\left(R_{1}, \ldots, R_{K}\right)$ such that

$$
\sum_{k \in \mathcal{S}} R_{k} \leq \log _{2}\left|\boldsymbol{I}+\sum_{k \in \mathcal{S}} \boldsymbol{H}_{k} \boldsymbol{\Psi}_{k} \boldsymbol{H}_{k}^{H}\right| \quad \text { for all } \mathcal{S} \subseteq\{1,2, \ldots, K\}
$$

for some $\boldsymbol{\Psi}_{1}, \ldots \boldsymbol{\Psi}_{K} \succeq \mathbf{0}$ with $\operatorname{tr}\left\{\boldsymbol{\Psi}_{k}\right\} \leq 1$, for $k=1, \ldots, K$. 
The sum-capacity of the Gaussian MIMO MAC is the optimal value of

$$
\begin{gathered}
\text { maximize } \log _{2}\left|\boldsymbol{I}+\sum_{k=1}^{K} \boldsymbol{H}_{k} \boldsymbol{\Psi}_{k} \boldsymbol{H}_{k}^{H}\right| \\
\text { subject to } \operatorname{tr}\left\{\boldsymbol{\Psi}_{k}\right\} \leq 1, \quad k=1, \ldots, K, \\
\boldsymbol{\Psi}_{k} \succeq \mathbf{0}, \quad k=1, \ldots, K .
\end{gathered}
$$

We can solve (3.3)-(3.5) by using the iterative water-filling algorithm of [32].

\subsubsection{The Special Case of $K=1$}

For $K=1$, the MIMO MAC reduces to a point-to-point MIMO link and the optimization problem (3.3)-(3.5) for finding the capacity reduces to

$$
\begin{aligned}
& \text { maximize } \log _{2}\left|\boldsymbol{I}+\boldsymbol{H}_{1} \boldsymbol{\Psi}_{1} \boldsymbol{H}_{1}^{H}\right| \\
& \text { subject to } \operatorname{tr}\left\{\boldsymbol{\Psi}_{1}\right\} \leq 1 \text { and } \boldsymbol{\Psi}_{1} \succeq \mathbf{0} .
\end{aligned}
$$

We solve (3.6)-(3.7) by applying the singular value decomposition to $\boldsymbol{H}_{1}$ and performing water-filling to find the optimal eigenvalues of $\boldsymbol{\Psi}_{1}$ [33].

\subsubsection{The Special Case of Gaussian SIMO MAC}

For the case of the Gaussian SIMO MAC, i.e., we have single-antenna transmitters, the channel matrix $\boldsymbol{H}_{k}$ reduces to the vector $\boldsymbol{h}_{k}$ for $k=1, \ldots, K$ and we define the channel matrix $\boldsymbol{H} \triangleq\left[\boldsymbol{h}_{1}, \ldots, \boldsymbol{h}_{k}\right] \in \mathbb{C}^{n_{r} \times K}$. Moreover, the transmit covariance matrix $\boldsymbol{\Psi}_{k}$ reduces to the scalar $\psi_{k} \geq 0$ for $k=1, \ldots, K$ and we define the vector $\boldsymbol{\psi} \triangleq\left[\psi_{1}, \ldots, \psi_{k}\right]^{T}$. Then, the optimization problem $(3.3)-(3.5)$ is written as

$$
\begin{aligned}
& \text { maximize } \log _{2}\left|\boldsymbol{I}+\boldsymbol{H} \operatorname{diag}\{\boldsymbol{\psi}\} \boldsymbol{H}^{H}\right| \\
& \text { subject to } \mathbf{0} \leq \boldsymbol{\psi} \leq \mathbf{1}
\end{aligned}
$$

It is straightforward to prove that optimal value of (3.8)-(3.9) is $\log _{2}\left|\boldsymbol{I}+\boldsymbol{H} \boldsymbol{H}^{H}\right|$ which is obtained for $\boldsymbol{\psi}=\mathbf{1}$. 


\subsubsection{The Special Case of Two-User Gaussian SISO MAC}

As a special case of the Gaussian MIMO MAC, we give the two-user Gaussian SISO MAC. The matched-filtered, symbol-sampled complex baseband signal at the receiver is

$$
y=h_{1} x_{1}+h_{2} x_{2}+e
$$

where $h_{1}$ and $h_{2}$ denote the complex scalar channels to the receiver from transmitters 1 and 2, respectively. All other quantities of (3.10) are defined similar to in (3.1). The capacity region of the SISO MAC is a pentagon in the first orthant and is given by the following theorem in [29] and [30].

Theorem 2. The capacity region of the Gaussian SISO MAC is the set of rate points $\left(R_{1}, R_{2}\right)$ such that

$$
\begin{aligned}
R_{1} & \leq \log _{2}\left(1+\left|h_{1}\right|^{2}\right) \\
R_{2} & \leq \log _{2}\left(1+\left|h_{2}\right|^{2}\right) \\
R_{1}+R_{2} & \leq \log _{2}\left(1+\left|h_{1}\right|^{2}+\left|h_{2}\right|^{2}\right) .
\end{aligned}
$$

The capacity region given in Theorem 2 is illustrated in Fig. 3.3. The corner points $\mathrm{A}$ and $\mathrm{B}$ are achieved when both transmitters use maximum power and the receiver performs successive decoding of the transmitters' messages. At point $\mathrm{A}$, the receiver first decodes the message from transmitter 1 by treating the signal received from transmitter 2 as additive noise. Therefore, transmitter 1 can at most achieve the rate

$$
R_{1, A}=\log _{2}\left(1+\frac{\left|h_{1}\right|^{2}}{\left|h_{2}\right|^{2}+1}\right) .
$$

The receiver can now decode the message from transmitter 2 by first subtracting the message from transmitter 1 from the received signal, which then consists of only the intended signal from transmitter 2 and the additive noise. Error free decoding can be achieved if

$$
R_{2, A}=\log _{2}\left(1+\left|h_{2}\right|^{2}\right) .
$$

Note that the point $\left(R_{1, A}, R_{2, A}\right)$ is the intersection between the lines $R_{2}=$ $\log _{2}\left(1+\left|h_{2}\right|^{2}\right)$ and $R_{1}+R_{2}=\log _{2}\left(1+\left|h_{1}\right|^{2}+\left|h_{2}\right|^{2}\right)$. Similar to point A, point $\mathrm{B}$ can be achieved by switching the order of decoding. The points on the straight line between the points A and B can be achieved by timesharing. That is, for a fraction $\tau$ of the time the MAC uses the encoding 
and decoding strategies achieving point $\mathrm{A}$ and for the other $(1-\tau)$ fraction it uses the strategies achieving point B. By varying $\tau \in[0,1]$, all points on the line of slope -1 can be achieved. The points on this line can also be achieved by simultaneous decoding of the two messages [34].

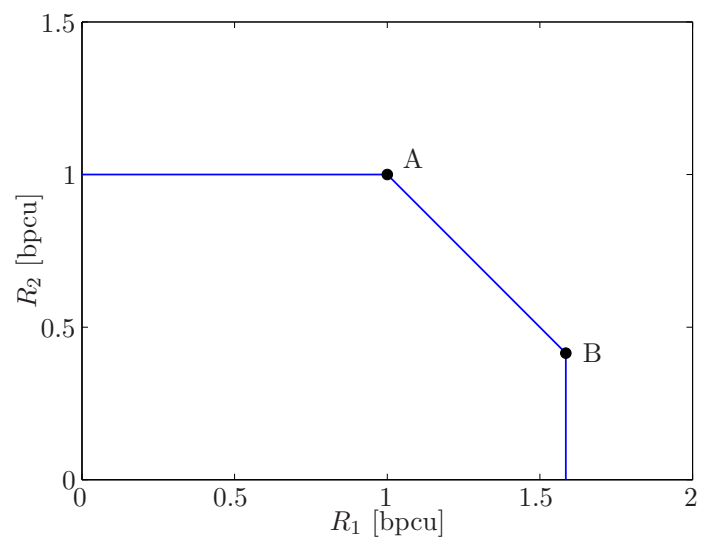

Figure 3.3: Capacity region of the two-user Gaussian SISO MAC with $\left|h_{1}\right|^{2}=2$ and $\left|h_{2}\right|^{2}=1$.

\subsection{Broadcast Channel}

The $\mathrm{BC}$ is a setup where a single transmitter communicates with $K \geq 2$ receivers simultaneously and in the same frequency band. The transmitter sends independent messages to the receivers which will see a signal that is a superposition of their intended signals and the signals intended for the other receivers, i.e., the unintended signals appear as interference. The receivers do not cooperate in the decoding of the messages. The receivers also experience some additive noise that models the thermal noise or, potentially, some unknown interference. In Fig. 3.4, we illustrate the MIMO BC.

The capacity region of the general BC still remains unknown. However, for the class of so-called stochastically degraded BCs (see, e.g., [35, Ch. 5.4] for a defintion), the capacity region is known. This BC was first introduced in [36] where the charactarization of its capacity region was conjectured. 


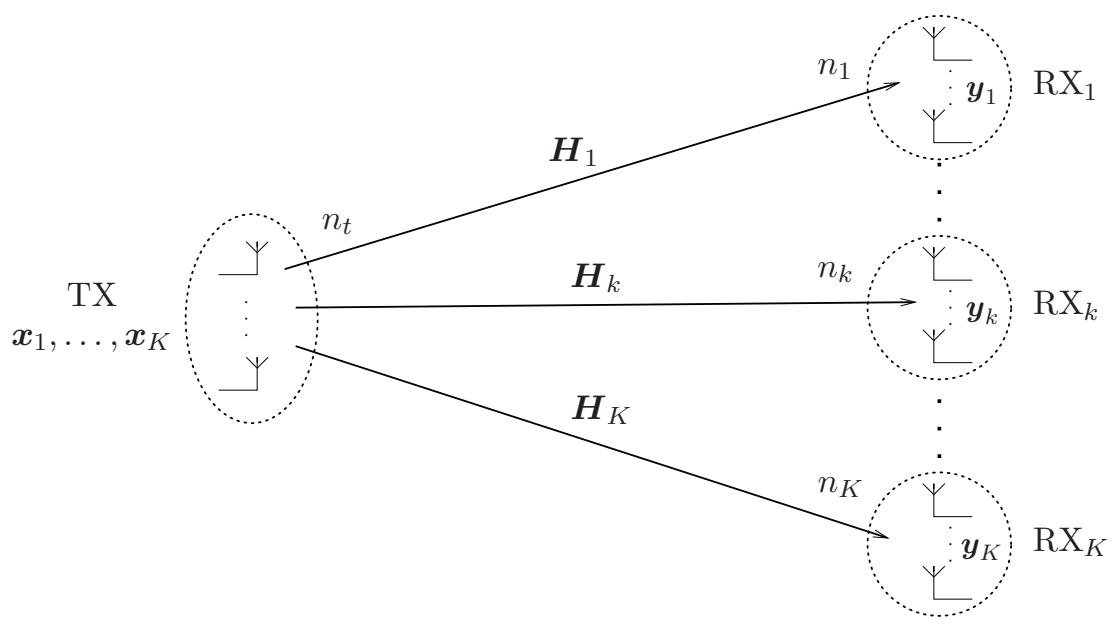

Figure 3.4: Illustration of the MIMO BC.

Later, the achievability and the converse of this region were established in [37] and [38], respectively. Fortunately, the Gaussian SISO BC is in general a stochastically degraded $\mathrm{BC}$ and the transmission technique that achieves the capacity is so-called superposition coding. The Gaussian MIMO $\mathrm{BC}$ is not degraded, but its capacity region is known. The sum-capacity of the MISO BC was established by [39], the sum-capacity of the general MIMO BC was given independently in [40-42], and the capacity region of the Gaussian MIMO BC were established in [43]. A key technique that enabled the establishment of the capacity of the Gaussian MIMO BC is the so-called dirty paper-coding (DPC), see [44].

We will now proceed by giving the capacity region of the Gaussian MIMO BC. We assume that the transmitter is equipped with $n_{t}$ antennas and receiver $k$ is equipped with $n_{k}$ antennas. Then, we let $\boldsymbol{H}_{k} \in \mathbb{C}^{n_{k} \times n_{t}}$ denote the channel matrix between the transmitter and receiver $k$. By $\boldsymbol{e}_{k} \sim \mathcal{C N}(\mathbf{0}, \boldsymbol{I})$, $k=1, \ldots, K$, we denote the additive i.i.d. Gaussian noise vectors. Moreover, the transmitted symbol intended for receiver $k$ is denoted by the vector $\boldsymbol{x}_{k}$ with covariance matrix $\boldsymbol{\Psi}_{k}$. These covariance matrices are subject to a normalized sum-power constraint, namely $\sum_{k=1}^{K} \mathrm{E}\left\{\left\|\boldsymbol{x}_{k}\right\|^{2}\right\}=\sum_{k=1}^{K} \operatorname{tr}\left\{\boldsymbol{\Psi}_{k}\right\} \leq$ 1. The matched-filtered, symbol-sampled complex baseband signal at the 
$k$ th receiver is

$$
\boldsymbol{y}_{k}=\sum_{l=1}^{K} \boldsymbol{H}_{k} \boldsymbol{x}_{l}+\boldsymbol{e}_{k} .
$$

The DPC is a non-linear transmission scheme that pre-cancels the interference using the fact that the transmitter knows all the messages and hence, the interference at the receivers, non-causally. The dual MAC is obtained by considering a Gaussian MIMO MAC where the channel between the $k$ th transmitter and the receiver is $\boldsymbol{H}_{k}^{H}$ and there is a sum-power constraint instead of individual power constraints. From the BC-MAC duality it follows that the achievable rate region of the DPC region is identical to the capacity region of the dual MAC under the same sum-power constraint. Consider an ordering $\pi$ on $\{1, \ldots, K\}$ of the receivers. The message intended for receiver $\pi(1)$ is encoded without considering the signals to the other receivers. Hence, all other signals appear as interference at receiver $\pi(1)$, i.e.,

$$
\boldsymbol{y}_{\pi(1)}=\boldsymbol{H}_{\pi(1)} \boldsymbol{x}_{\pi(1)}+\underbrace{\sum_{l=2}^{K} \boldsymbol{H}_{\pi(1)} \boldsymbol{x}_{\pi(l)}}_{\text {interference }}+\boldsymbol{e}_{\pi(1)} \text {. }
$$

When the transmitter encodes the message for receiver $\pi(k)$, it knows the signal intended for receivers $\pi(1), \ldots \pi(k-1)$. Hence, these signals are canceled out by the DPC and, hence, receiver $\pi(k)$ only experiences the signals intended for receivers $\pi(k+1), \ldots \pi(K)$ as interference. Therefore, the signal at receiver $\pi(k)$ is modeled as

$$
\boldsymbol{y}_{\pi(k)}=\boldsymbol{H}_{\pi(k)} \boldsymbol{x}_{\pi(k)}+\sum_{l=k+1}^{K} \boldsymbol{H}_{\pi(k)} \boldsymbol{x}_{\pi(l)}+\boldsymbol{e}_{k} .
$$

For a set of transmit covariance matrices $\left\{\boldsymbol{\Psi}_{\pi(1)}, \ldots, \boldsymbol{\Psi}_{\pi(K)}\right\}$, user $\pi(k)$ achieves the rate

$$
R_{\pi(k)} \leq \log _{2} \frac{\left|\sum_{l=k}^{K} \boldsymbol{H}_{\pi(k)} \boldsymbol{\Psi}_{\pi(l)} \boldsymbol{H}_{\pi(k)}^{H}+\boldsymbol{I}\right|}{\left|\sum_{l=k+1}^{K} \boldsymbol{H}_{\pi(k)} \boldsymbol{\Psi}_{\pi(l)} \boldsymbol{H}_{\pi(k)}^{H}+\boldsymbol{I}\right|} .
$$

The following theorem [43] gives the capacity region of the K-user Gaussian MIMO BC. 
Theorem 3. The capacity region of the $K$-user Gaussian MIMO BC is the convex hull of the set of rate points $\left(R_{1}, \ldots, R_{K}\right)$ such that $(3.17)$ is satisfied for some ordering $\pi$ on $\{1, \ldots, K\}$ and a set of transmit covariance matrices $\boldsymbol{\Psi}_{1}, \ldots, \boldsymbol{\Psi}_{K} \succeq \mathbf{0}$ with $\sum_{k=1}^{K} \operatorname{tr}\left\{\boldsymbol{\Psi}_{k}\right\} \leq 1$.

For the computation of the sum-capacity of the Gaussian MIMO BC, (3.17) is not that useful. The function is not concave in the transmit covariance matrices $\boldsymbol{\Psi}_{1}, \ldots, \boldsymbol{\Psi}_{K}$ and we have to consider all possible orderings of the receivers. Instead, the sum-capacity can be computed using the BC MAC duality [40]. Then, we solve the optimization problem

$$
\begin{array}{ll}
\text { maximize } & \log _{2}\left|\sum_{k=1}^{K} \boldsymbol{H}_{k}^{H} \boldsymbol{\Psi}_{k} \boldsymbol{H}_{k}+\boldsymbol{I}\right| \\
\text { subject to } & \sum_{k=1}^{K} \operatorname{tr}\left\{\boldsymbol{\Psi}_{k}\right\} \leq 1, \\
\boldsymbol{\Psi}_{k} \succeq \mathbf{0}, \quad k=1, \ldots, K .
\end{array}
$$

Since the optimal function (3.18) is concave in the variables and the constraints (3.19)-(3.20) are convex, the problem (3.18)-(3.20) is convex and can be solved efficiently.

\subsubsection{The Special Case of Gaussian MISO BC}

For the case of the Gaussian MISO BC, i.e., single-antenna receivers, the channel matrix $\boldsymbol{H}_{k}$ reduces to the row vector $\boldsymbol{h}_{k}^{T}$ for $k=1, \ldots, K$ and we define the channel matrix $\boldsymbol{H} \triangleq\left[\boldsymbol{h}_{1}, \ldots, \boldsymbol{h}_{k}\right]^{T} \in \mathbb{C}^{K \times n_{t}}$. Moreover, the transmit covariance matrix $\boldsymbol{\Psi}_{k}$ reduces to the scalar $\psi_{k} \geq 0$ for $k=1, \ldots, K$ and we define the vector $\boldsymbol{\psi} \triangleq\left[\psi_{1}, \ldots, \psi_{k}\right]^{T}$. Then, the optimization problem (3.18)-(3.19) is written as

$$
\begin{aligned}
& \operatorname{maximize} \log _{2}\left|\boldsymbol{I}+\boldsymbol{H}^{H} \operatorname{diag}\{\boldsymbol{\psi}\} \boldsymbol{H}\right| \\
& \text { subject to } \mathbf{1}^{T} \boldsymbol{\psi} \text { and } \boldsymbol{\psi} \geq \mathbf{0} .
\end{aligned}
$$

Since (3.8)-(3.9) is a convex problem in the variable $\boldsymbol{\psi}$, the objective value can be computed efficiently. 


\subsubsection{The Special Case of Two-User Gaussian SISO BC}

For the two-user Gaussian SISO BC, we have the received signal model

$$
\left\{\begin{array}{l}
y_{1}=h_{1} x_{1}+h_{1} x_{2}+e_{1} \\
y_{2}=h_{2} x_{2}+h_{2} x_{1}+e_{2}
\end{array}\right.
$$

where all quantities are scalar versions of the ones defined for (3.14). By assuming $\left|h_{1}\right|>\left|h_{2}\right|$ (or alternatively $\left|h_{1}\right|<\left|h_{2}\right|$ ), we have a stochastically degraded $\mathrm{BC}$ and receiver 1 can always decode the message intended for receiver 2 before it decodes its own message. To see this, we assume that the transmitter allocates the normalized power $P$ to receiver 1 and $1-P$ to receiver 2 and observe that receiver 2 can decode its message, treating the signal intended for receiver 1 as noise, whenever the rate is upper bounded as

$$
R_{2} \leq \log _{2}\left(1+\frac{\left|h_{2}\right|^{2}(1-P)}{1+\left|h_{2}\right|^{2} P}\right)=\log _{2}\left(1+\frac{1-P}{1 /\left|h_{2}\right|^{2}+P}\right) .
$$

On the other hand receiver 1 can decode the message for receiver 2 , treating its own intended signal as noise

$$
R_{2} \leq \log _{2}\left(1+\frac{\left|h_{1}\right|^{2}(1-P)}{1+\left|h_{1}\right|^{2} P}\right)=\log _{2}\left(1+\frac{1-P}{1 /\left|h_{1}\right|^{2}+P}\right) .
$$

Clearly, the right-hand-side of (3.25) is larger than that of (3.24). Once receiver 1 has decoded the interference, it substracts it and obtains an interference free signal and can achieve the rate

$$
R_{1} \leq \log \left(1+\left|h_{1}\right|^{2} P\right)
$$

This discussion leads to the following theorem for the capacity region of the Gaussian SISO BC:

Theorem 4. The capacity region of the Gaussian SISO BC is the set of rate pairs $\left(R_{1}, R_{2}\right)$ satisfying (3.24) and (3.26) for some $P \in[0,1]$.

\subsection{Interference Channel}

The $K$-user IC consists of $K$ transmitter-receiver pairs operating simultaneously in the same piece of spectrum. Transmitter $k$ wants to convey information only to receiver $k$ but it causes interference to the other receivers 


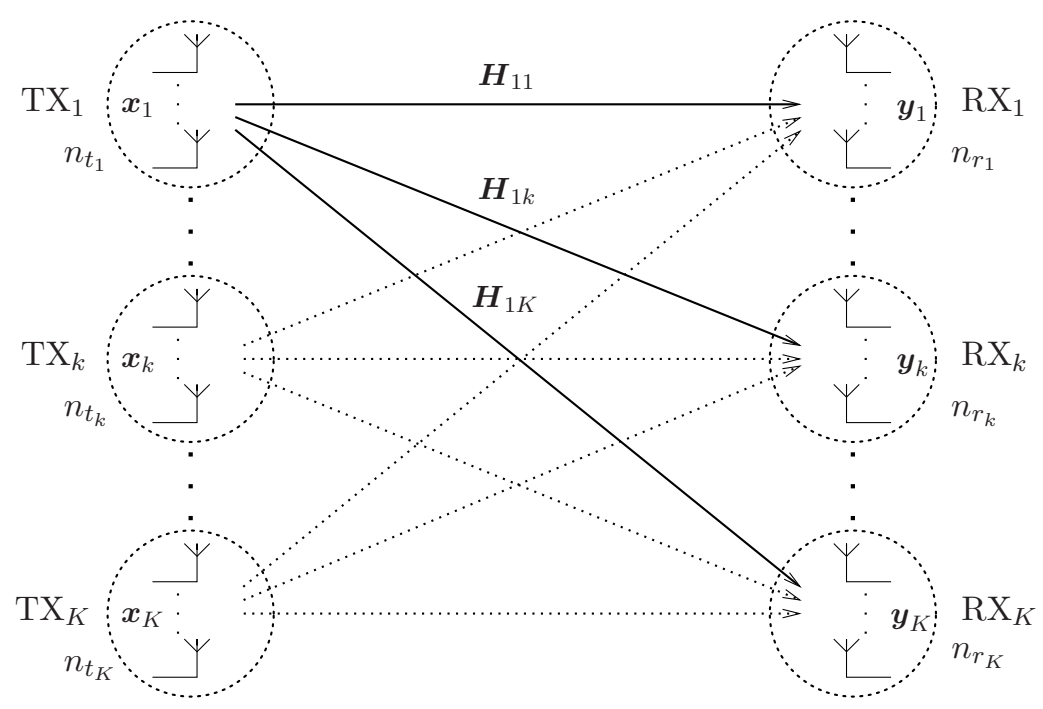

Figure 3.5: Illustration of the MIMO IC.

as well. The IC can be seen as a generalization of both the MAC and the $\mathrm{BC}$ since the transmission is from many transmitters to many receivers and there is no cooperation in encoding and decoding of the massages.

In Fig. 3.5, we illustrate the MIMO IC. Transmitter $j$ and receiver $k$ are equipped with $n_{t_{j}}$ and $n_{r_{k}}$ antennas, respectively, and the channel between them is denoted by $\boldsymbol{H}_{j k}$ which is a complex $n_{r_{k}} \times n_{t_{l}}$ matrix. The matchedfiltered, symbol-sampled complex baseband signal at the $k$ th receiver is

$$
\boldsymbol{y}_{k}=\boldsymbol{H}_{k k} \boldsymbol{x}_{k}+\sum_{j=1, j \neq k}^{K} \boldsymbol{H}_{j k} \boldsymbol{x}_{j}+\boldsymbol{e}_{k}
$$

By $\boldsymbol{e}_{k} \sim \mathcal{C N}(\mathbf{0}, \boldsymbol{I}), k=1, \ldots, K$, we denote the additive i.i.d. Gaussian noise vectors. Moreover, the transmitted symbol intended for receiver $k$ is denoted by the vector $\boldsymbol{x}_{k}$ with covariance matrix $\boldsymbol{\Psi}_{k}$, which is subject to the normalized power constraint $\mathrm{E}\left\{\left\|\boldsymbol{x}_{k}\right\|^{2}\right\}=\operatorname{tr}\left\{\boldsymbol{\Psi}_{k}\right\} \leq 1$.

As a special case of the Gaussian MIMO IC in (3.27), we have the twouser Gaussian SISO IC, where the matched-filtered, symbol-sampled com- 
plex baseband signals at receivers are

$$
\left\{\begin{array}{l}
y_{1}=h_{11} x_{1}+h_{21} x_{2}+e_{1} \\
y_{2}=h_{22} x_{2}+h_{12} x_{1}+e_{2}
\end{array}\right.
$$

where $h_{j k}$ is the scalar complex channel between transmitter $j$ and receiver $k$. All other quantities in (3.28) are defined in the same way as for the MIMO IC, but they are scalars instead of vectors.

The IC has been subject to a substantial amount of research during the last four decades. Despite that, the capacity region of the IC still remains unknown in general. Not even the capacity region of the two-user Gaussian SISO IC is known in general. The single-antenna IC was first studied in [45] where inner and outer bounds on the capacity were established. The best known inner bound of the SISO IC was established by [46]. To derive this bound, the authors used the ideas of rate splitting and simultaneous decoding. The idea is that the message is split into public and private parts. The public message is decoded by the unintended receivers whereas the private is not. Indeed, the capacity region of the two-user Guassian SISO IC is known for the special case of strong interference, i.e., $\left|h_{12}\right| \geq\left|h_{11}\right|$ and $\left|h_{21}\right| \geq\left|h_{22}\right|$. This capacity region, which was independently established in [46] and [47], is obtained by using Gaussian coding and non-unique simultaneous decoding. That is, each receiver tries to decode both messages, but unintended messages do not have to be correctly decoded. For the case of weak interference, i.e.,

$$
\begin{aligned}
& \left|h_{21}\right|^{2}\left(1+\left|h_{12}\right|^{2}\right)^{2} /\left|h_{22}\right|^{2} \leq \rho_{2}\left(1-\rho_{1}\right), \\
& \left|h_{12}\right|^{2}\left(1+\left|h_{21}\right|^{2}\right)^{2} /\left|h_{11}\right|^{2} \leq \rho_{1}\left(1-\rho_{2}\right)
\end{aligned}
$$

for some $\rho_{1}, \rho_{2} \in[0,1]$, the sum-capacity is known to be achieved by Gaussian coding and treating interference as noise, e.g., see [48]. A genie-based outer bound of the capacity region of the Gaussian SISO IC was given in [49]. In [50], it was proven that any point on the boundary of the achievable region of [46] differs from the capacity region by no more than one bit per component. That is, if $\left(R_{1}, R_{2}\right)$ lies on the boundary of the achievable region, then $\left(R_{1}+1, R_{2}+1\right)$ lies outside the capacity region. The proof was established using the outer bound given in [49].

For the general $K$-user Gaussian SISO IC, we have even less understanding of the capacity region. The best knowledge is obtained by interference alignment where the transmitters align the interference to a subspace of the 
receivers' signal spaces. These subspaces are discarded and the intended signals are reconstructed from the orthogonal subspaces. Interference alignment is a useful tool to determine the achievable number of DoF of an interference channel. For example, the $K$-user SISO IC with time-varying channels achieves $K / 2 \mathrm{DoF}$ [51]. Also for the MIMO IC, interference alignment is one of very few methods to understand its behavior. In [51] it was proven that the Gaussian MIMO IC achieves at least $\sum_{k=1}^{K} \min \left\{n_{t_{k}}, n_{r_{k}}\right\} / 2$ DoF. Similarly to the Gaussian SISO IC, the capacity region of the two-user MIMO IC has be characterized to within a constant gap, which depends on the number of receiver antennas [52]. Namely, if $\left(R_{1}, R_{2}\right)$ lies on the boundary of the achievable region, which is an extension of that in [46], then $\left(R_{1}+1, R_{2}+1\right)$ lies outside the capacity region. 


\section{Chapter 4}

\section{Contributions of the Thesis and Open Research Directions}

In this chapter we briefly describe the contributions of this thesis and put the results into a greater context. By doing so, we also point out some future research directions. The contributions of this thesis consist of two parts. The first part focuses on achievable rate regions of the two-user MISO IC. The second part considers resource allocation in cellular networks. Here the focus is on the non-orthogonal spectrum sharing.

\subsection{Achievable Rate Regions}

This first part considers definitions, characterizations, and efficient computations of achievable rate regions for the two-user MISO IC. Depending on the kind of CSI and the behavior of fading channels, we study different achievable rate regions.

Paper A considers the instantaneous rate region for the case where the receivers are able to perform successive interference cancellation (SIC). The corresponding rate region has previously been characterized in [53] for the case where the RXs treat interference as noise and in [54] for the case of SIC. 
In these previous works, the focus was on parameterizations of the beamforming vectors in order to reduce the search space for beamforming vector pairs which yield Pareto-optimal (PO) points. A point is $\mathrm{PO}$ if it is not possible to increase the rate of one user without decreasing the rate of the other user. These points lie on the outer (north-east) boundary of the rate region, which is called the Pareto Boundary. Here, we utilize the parameterizations of [53] and [54] to formulate scalar, quasi-concave, optimization problems which very efficiently find the pair of PO beamforming vectors.

In Papers B and C, we consider the case of fast fading with statistical CSI. For this scenario it makes sense to study the ergodic rate region. We characterize the transmit covariance matrices which separately fulfill the necessary conditions for being PO. These works extend the results for the instantaneous rate region in [53] to the ergodic rate region. Still, these contributions were among the first on the ergodic rate region for the MISO IC.

In Paper D, we consider the case of slow-fading, for which it makes sense to study outage rate regions, i.e., which rate pairs can be achieved with a certain probability of outage, for both instantaneous and statistical CSI. We provide four different definitions of the outage rate regions, which depend on whether there is instantaneous or statistical CSI and whether we study individual or common outage events. Common outage is declared when at least one of the users is in outage. Individual outage considers each user separately. The provideddefinitions are novel for the IC, but extend those for the BC and MAC, e.g., in [57] and [58].

For the MISO interference channel, there are several recent publications on characterizations and efficient computations of various achievable rate regions. These assume either instantaneous or statistical CSI, either instantaneous, ergodic, or outage rate regions, with or without successive SIC. Another, related, topic is the instantaneous rate region with robust (worstcase) beamforming for imperfect CSI without SIC studied in [56]. The early works, if there exist any, that deal with fundamental aspects of the MISO IC for combinations of these assumptions are summarized in Tab. 4.1. As it can be seen, SIC has only been considered for instantaneous rate regions. For all other scenarios, the achievable rate regions and characterizations of them are open problems. However, for outage rate regions with instantaneous CSI, we believe that is straightforward to include SIC, since the definitions do not restrict to the case of treating interference as noise. For all the other cases, we expect that it is quite hard to incorporate SIC. 


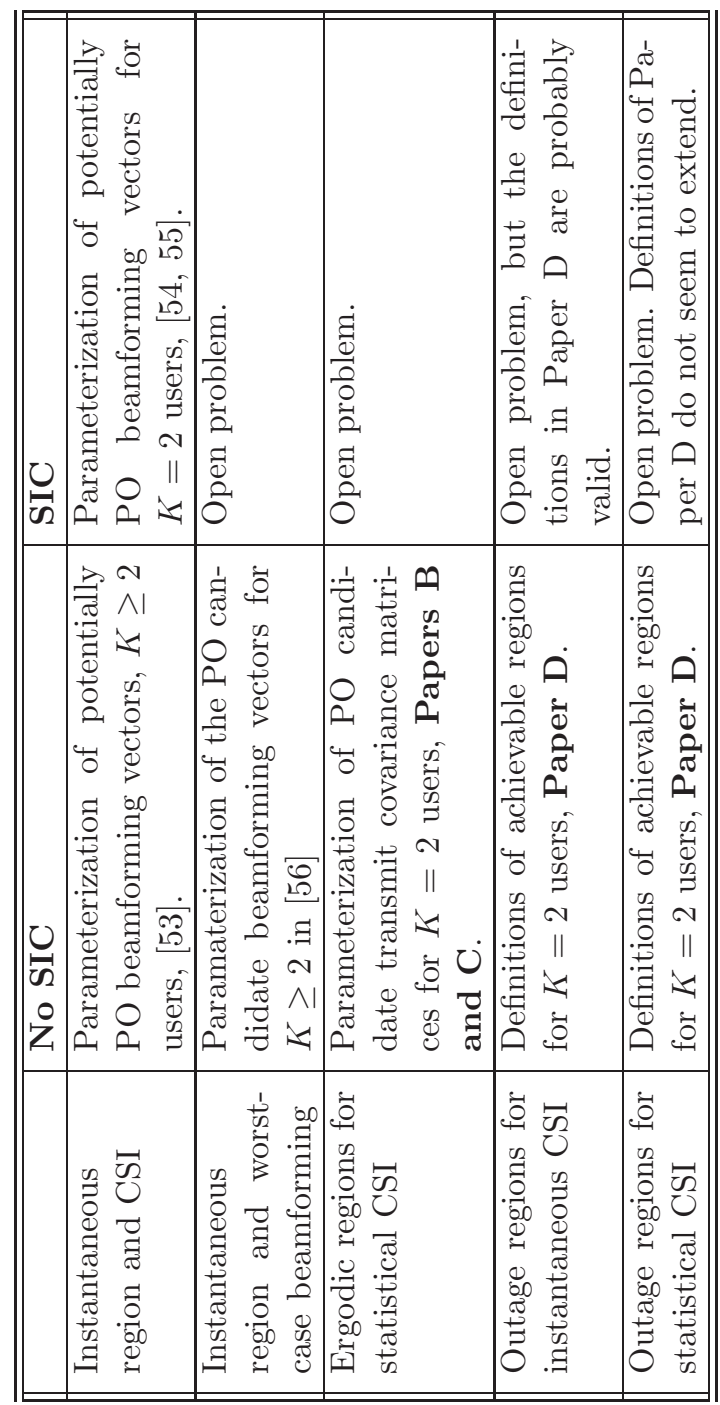

Table 4.1: Summary of contributions to the understanding of the MISO interference channel. 
Once we have gained a fundamental understanding of the various achievable rate regions for the MISO interference channel, we want to find computationally efficient methods to determine the outer boundary of each region. The existing contributions on this problem are summarized in Tab. 4.2. We only have very efficient methods for the instantaneous rate region for the two-user MISO IC with and without SIC. For the ergodic and outage rate regions with statistical CSI there is a need to compute the PO candidate transmit covariance matrices separately. For the robust, worst-case beamforming problem and the statistical CSI regions where we restrict to single-stream beamforming, the PO candidate beamforming vectors can be computed via the so-called interference levels. For the robust beamforming case, we solve a second-order cone program (SOCP), [56] and for the statistical CSI regions, we solve a semi-definite program (SDP), [59]. Once all PO candidate beamforming vectors or transmit covariance matrices are computed, we need to perform a brute-force search over all pairs to find the Pareto boundary. For ergodic and outage rate regions with statistical CSI, multistream beamforming is optimal in general. For these scenarios, we have more than one real parameter per transmit covariance matrix, in general. For the outage rate regions with instantaneous CSI, we have to compute the probability that a rate point lies in a random instantaneous rate region. For this problem, we are not aware of any closed-form method and to estimate this probability, we use Monte-Carlo simulations.

For the case of two transmitter-receiver pairs (or users) with single-stream beamforming, for all cases of CSI knowledge but without SIC, it is feasible to compute the boundaries via the parameters and a brute-force comparison of all combinations of the beamforming vectors. For the case of general $K \geq 2$ users without SIC capability, we need $K(K-1)$ parameters to find all PO candidate beamforming vectors, see [56] and [60]. Even for $K=3$ it is a quite complex task to compute the Pareto boundary. The most efficient method known to us for computing the boundary of the instantanous rate region for the $K$-user MISO IC without SIC is to use so-called rate profiles combined with SOCPs, [61]. For the problem of SIC, the complexity of finding the boundary grows as $\mathcal{O}\left(((K-1) !)^{K}\right)$, see the discussion in Sec. 8 of Paper A.

This discussion illustrates that there are many open problems left to consider for the MISO IC. For the two-user case we believe that the most important problem is to incorporate SIC into the ergodic and outage rate regions. For the $K$-user MISO IC the first step should be towards definition and efficient computations of the ergodic and outage rate regions without SIC. Another problem which we did not touch upon in this thesis is the MISO IC with 


\begin{tabular}{|c|c|c|c|c|c|c|c|}
\hline$\frac{U}{\sqrt{2}}$ & 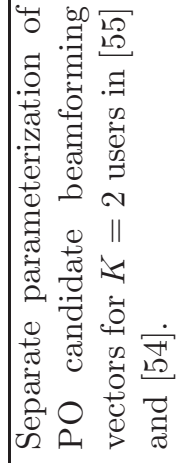 & 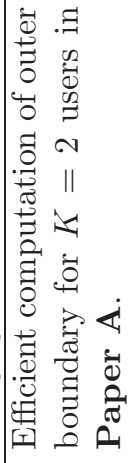 & 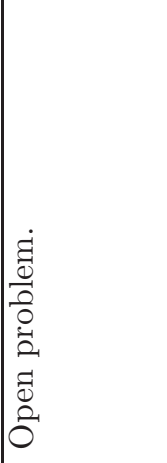 & 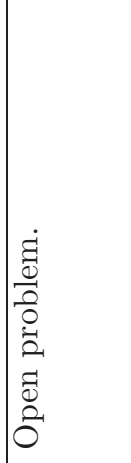 & 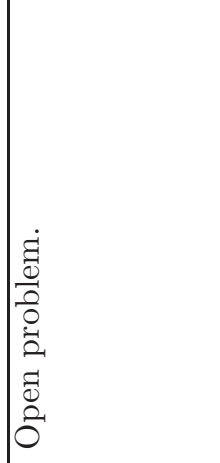 & 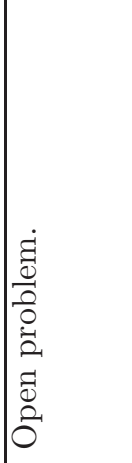 & 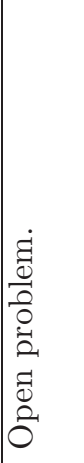 \\
\hline $\begin{array}{l}0 \\
\text { U } \\
0 \\
Z \\
Z\end{array}$ & 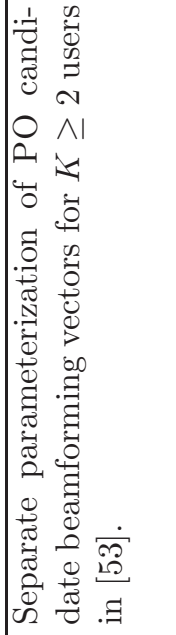 & 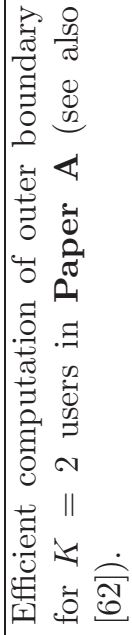 & 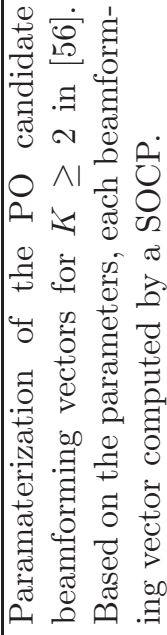 & 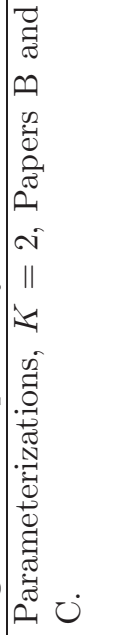 & 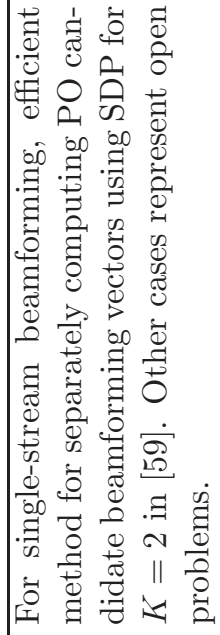 & 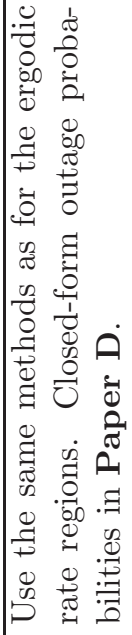 & 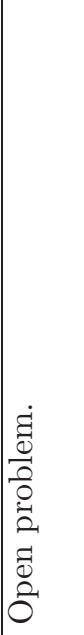 \\
\hline & 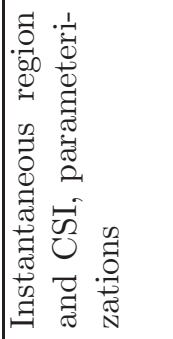 & 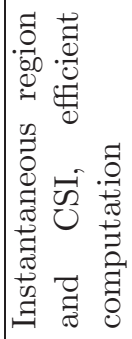 & 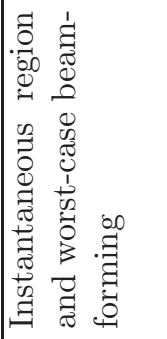 & 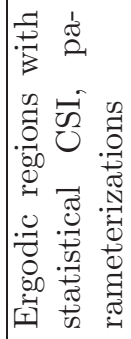 & 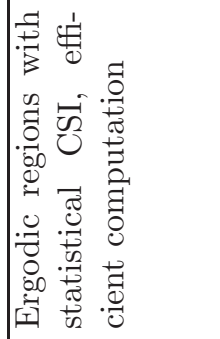 & 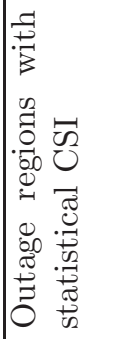 & 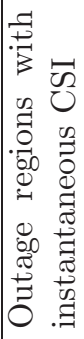 \\
\hline
\end{tabular}

Table 4.2: Contributions on efficient computations of achievable rate regions. 
multiple subchannels with a total power constraint. What do the regions look like then and can we potentially utilize any correlation between the channels in the computation of the rate region? However, we expect that this is also a quite complicated problem, which can be solved numerically, but which does not provide much of analytic insights.

\subsection{Resource Allocation and Spectrum Sharing}

In this part, we consider the resource allocation, mainly user-selection, problem and spectrum sharing.

In Paper E, we study a small network of two co-located transmitters. This setup corresponds to the inter-operator spectrum sharing scenario. We use upper and lower bounds on the sum-capacity of the MISO BC and the MISO $\mathrm{IC}$ in order to investigate if there is something to gain from spectrum sharing when it is not possible to perform user selection. That is, we cannot exploit multi-user diversity to increase the rates. This corresponds to a delay intolerant scenario where some higher-level scheduler has already selected the users. In this case we see no gain by performing non-orthogonal spectrum sharing. If one operator has resources left, they should first be used for orthogonal spectrum sharing.

In Paper F, we consider the problem of proportional fair scheduling (PFS) for the downlink of a non-orthogonal spectrum sharing setup of two multiantenna TXs and many RXs per TX. The PFS is implemented using weighted sum-rate (WSR) maximization, which is NP-hard in general. Hence suboptimal methods, e.g., iterative weighted minimum mean-square error approaches, are needed to obtain solutions which are reasonable, [63]. However these methods still have high computational complexity and they require overhead communication between transmitters and receivers as well as between transmitters. Therefore, we propose two heuristic joint beamforming and user selection algorithms. First the algorithm selects users based on simple beamforming schemes. Once the users are selected, the algorithm uses optimal beamforming vectors to maximize the rates.

In both Papers E and F, we restrict to the case of only two TXs serving at most one RX per subchannel. Also, there is no optimization of the power allocation across the subchannels. For these cases we can utilize the efficient method in Paper A to compute the boundary of the rate region, and hence 
the maximum (weighted) sum-rate. If we relax the aforementioned limitations, we do not have computationally efficient optimal methods any longer. Here, there are opportunities for future research.

The broader area of non-orthogonal spectrum sharing versus orthogonal spectrum sharing should be examined for more general setups, including traffic modeling. Also overhead due to coordination between TXs and acquiring CSI should be taken into account. In this way one could examine if the gains from non-orthogonal spectrum sharing over orthogonal spectrum sharing are worth striving for in practice.

\subsection{Included Publications}

\section{Paper A: Efficient Computation of Pareto Optimal Beamforming Vectors for the MISO Interference Channel with Successive Inter- ference Cancellation}

Authored by J. Lindblom, E. Karipidis, and E. G. Larsson.

Published in the IEEE Transactions on Signal Processing, Oct. 2013.

We study the two-user multiple-input single-output (MISO) Gaussian interference channel where the transmitters have perfect channel state information and employ single-stream beamforming. The receivers are capable of performing successive interference cancellation, so when the interfering signal is strong enough, it can be decoded, treating the desired signal as noise, and subtracted from the received signal, before the desired signal is decoded. We propose efficient methods to compute the Pareto-optimal rate points and corresponding beamforming vector pairs, by maximizing the rate of one link given the rate of the other link. We do so by splitting the original problem into four subproblems corresponding to the combinations of the receivers' decoding strategies - either decode the interference or treat it as additive noise. We utilize recently proposed parameterizations of the optimal beamforming vectors to equivalently reformulate each subproblem as a quasi-concave problem, which we solve very efficiently either analytically or via scalar numerical optimization. The computational complexity of the proposed methods is several orders-of-magnitude less than the complexity of the state-of-the-art methods. We use the proposed methods to illustrate the 
effect of the strength and spatial correlation of the channels on the shape of the rate region.

Paper B: Parameterization of the MISO IFC Rate Region: The Case of Partial Channel State Information

Authored by J. Lindblom, E. G. Larsson, and E. A. Jorswieck.

Published in the IEEE Transactions on Wireless Communications, Feb. 2010.

We study the achievable rate region of the multiple-input single-output (MISO) interference channel (IFC), under the assumption that all receivers treat the interference as additive Gaussian noise. We assume the case of two users, and that the channel state information (CSI) is only partially known at the transmitters. Our main result is a characterization of Pareto-optimal transmit strategies, for channel matrices that satisfy a certain technical condition. Numerical examples are provided to illustrate the theoretical results.

\section{Paper C: Selfishness and Altruism on the MISO Interference Chan- nel: The Case of Partial Transmitter CSI}

Authored by J. Lindblom, E. Karipidis, and E. G. Larsson.

Published in the IEEE Communication Letters, Sep. 2009.

We study the achievable ergodic rate region of the two-user multiple-input single-output interference channel, under the assumptions that the receivers treat interference as additive Gaussian noise and the transmitters only have statistical channel knowledge. Initially, we provide a closed-form expression for the ergodic rates and derive the Nash-equilibrium and zero-forcing transmit beamforming strategies. Then, we show that combinations of the aforementioned selfish and altruistic, respectively, strategies achieve Paretooptimal rate pairs.

\section{Paper D: Achievable Outage Rate Regions for the MISO Interfer- ence Channel}

Authored by J. Lindblom, E. Karipidis, and E. G. Larsson.

This is an extended version of a letter published in the IEEE Wireless Communication Letters, Aug. 2013. It is extended by some results presented 
at the Asilomar Conference on Signals, Systems, and Computers, 2009, and some unpublished materials.

We consider the slow-fading two-user multiple-input single-output (MISO) interference channel. We want to understand which rate points can be achieved, allowing a non-zero outage probability. We do so by defining four different outage rate regions. The definitions differ on whether the rates are declared in outage jointly or individually and whether the transmitters have instantaneous or statistical channel state information (CSI). The focus is on the instantaneous CSI case with individual outage, where we propose a stochastic mapping from the rate point and the channel realization to the beamforming vectors. A major contribution is that we prove that the stochastic component of this mapping is independent of the actual channel realization. For the case of statistical CSI, under the assumption of zero-mean Gaussian channel vectors, we compute the outage probabilities in closed form. We also prove that the statistical CSI outage rate regions are always contained in the instantaneous CSI outage rate regions and that the common outage rate regions are always contained in the individual outage rate regions.

\section{Paper E: Does Non-Orthogonal Spectrum Sharing in the Same Cell Improve the Sum-Rate of Wireless Operators?}

Authored by J. Lindblom and E. G. Larsson.

Published in the proceedings of The 13th IEEE International Workshop on Signal Processing Advances Wireless Commununications (SPAWC), 2012.

We study non-orthogonal spectrum sharing to determine under what circumstances operators can gain by such sharing. To model the spectrum sharing, we use the multiple-input single-output (MISO) interference channel (IC) assuming that the operators transmit in the same band. For the baseline scenario of no sharing, we use the MISO broadcast channel (BC) assuming that the operators transmit in disjunct bands. For both the IC and BC, we give achievable (lower) and upper bounds on the maximum sum-rate. While these bounds are well-known we also propose a new fast algorithm for finding a lower bound on the sum-rate of the BC using linear beamforming.

We use the bounds to numerically evaluate the potential gain of nonorthogonal spectrum sharing. In this study we assume that the operators efficiently utilize all their spatial degrees of freedom. We will see that the gains from spectrum sharing under these circumstances are limited. 


\section{Paper F: Joint User Selection and Beamforming Schemes for Inter- Operator Spectrum Sharing}

Authored by J. Lindblom, E. G. Larsson, and E. Karipidis.

Submitted to the IEEE International Conference on Acoustics, Speech, and Signal Processing (ICASSP), 2014.

We consider the downlink of an inter-operator spectrum sharing scenario where two operators share the same piece of spectrum and use it simultaneously. A base station of one operator cooperates with a base station of the other operator in order perform joint user selection and beamforming using a central unit. Optimal scheduling, in the sense of maximizing sum-rate or proportional fairness, is in many cases infeasible due to high computational complexity. Therefore, we propose a heuristic algorithm that schedules users based on simple beamforming techniques. Once the users are scheduled, we compute the optimal beamforming vectors for them. This method still performs an exhaustive user search. Therefore, we also propose a greedy user selection scheme. From numerical evaluations, we notice that these schemes perform close to the optimal selection. Also, we use our proposed methods to identify when spectrum sharing provides extra gains over the non-sharing scenario.

\subsection{Publications not Included in the Thesis}

The following publications contain work done by the author, but they are not included in this thesis.

J. Lindblom, E. G. Larsson, and E. A. Jorswieck, "Parameterization of the MISO Interference Channel with Transmit Beamforming and Partial Channel State Information," in Proc. Forty-Second Asilomar Conf. Signals, Systems Computers, Pacific Grove, CA, USA.

E. G. Larsson, E. Jorswieck, J. Lindblom, and R. Mochaourab, "Game theory and the flat fading Gaussian interference channel," IEEE Signal Processing Mag., vol. 26, no. 5, pp. 18-27, Sep. 2009. 
E. Karipidis, A. Gründinger, J. Lindblom, and E. G. Larsson, "Paretooptimal Beamforming for the MISO Interference Channel with Partial CSI," in Proc. 3rd IEEE Int. Workshop Computational Advances Multi-Sensor Adaptive Process. (CAMSAP), Aruba, Dutch Antilles, Dec. 2011, pp. 5-8.

J. Lindblom and E. Karipidis, "Cooperative Beamforming for the MISO Interference Channel," in Proc. European Wireless Conference (EW), Lucca, Italy, Apr. 2010, pp. 631-638.

J. Lindblom, E. Karipidis, and E. G. Larsson, "Closed-Form Parameterization of the Pareto Boundary for the Two-User MISO Interference Channel," in Proc. IEEE International Conference on Acoustics, Speech and Signal Processing (ICASSP), Prague, Czech Republic, May. 2011, pp. 3372-3375.

E. Karipidis, D. Gesbert, M. Haardt, K.-M. Ho, E. Jorswieck, E. G. Larsson, J. Li, J. Lindblom, C. Scheunert, M. Schubert, and N. Vucic, "Transmit Beamforming for Inter-Operator Spectrum Sharing," in Proc. Future Network 85 Mobile Summit (FutureNetw), Warsaw, Poland, Jun. 2011, pp. 1-8.

J. Lindblom, E. Karipidis, and E. G. Larsson, "Efficient Computation of the Pareto Boundary for the Two-User MISO Interference Channel with Multi-User Decoding Capable Receivers," in Proc. 4th IEEE Int. Workshop Computational Advances Multi-Sensor Adaptive Process. (CAMSAP), San Juan, Puerto Rico, Dec. 2011, pp. 241-244.

J. Luo, J. Lindblom, J. Li, R. Mochaourab, A. Kortke, E. Karipidis, M. Haardt, E. Jorswieck, and E. G. Larsson, "Transmit Beamforming for Inter-Operator Spectrum Sharing: From Theory to Practice," in Proc. Int. Symp. Wireless Commun. Systems (ISWCS), Paris, France, Aug. 2012, pp. 291-295.

R. Gangula, D. Gesbert, J. Lindblom, and E. G. Larsson, "On the Value of Spectrum Sharing among Operators in Multicell Networks" in Proc. Frth IEEE Veh. Tech. Conf. (VTC), Dresden, Germany, Jun. 2013. 
44 Chapter 4. Contributions of the Thesis and Open Research Directions 


\section{Bibliography}

[1] Cisco Systems Inc., "Cisco visual networking index: Global mobile data traffic forecast update, 2012-2017," 2013, technical White Paper.

[2] M. Lazarus, "The great spectrum famine," IEEE Spectr., vol. 47, no. 10, pp. 26-31, Oct. 2010.

[3] J. Mitola, "Cognitive radio for flexible mobile multimedia communications," in Proc. IEEE International Workshop on Mobile Multimedia Commun. (MoMuC), San Diego, CA, Nov. 1999, pp. 3-10.

[4] J. Peha, "Sharing spectrum through spectrum policy reform and cognitive radio," Proc. IEEE, vol. 97, no. 4, pp. 708-719, Apr. 2009.

[5] B. G. Mölleryd, "Mobile nework sharing - Swedish experiences," http://www.slideshare.net/Garry54/mobile-network-sharing-swedishexperiences, May 2010.

[6] Ericsson AB, "Spectrum sharing - fast-track capacity with licensed shared access," 2013, technical White Paper.

[7] E. A. Jorswieck, L. Badia, F. T, M. Haardt, E. Karipidis, J. Luo, and R. Pisz, "Resource sharing improves the network efficiency for network operators," SAPHYRE, 2011, White Paper 1, available at http://www.saphyre.eu/publications/.

[8] M. Szydelko, "D5.3: Business models, cost analysis and advices for spectrum policy and regulation for scenario I (spectrum sharing)," SAPHYRE, Jun. 2012, available at http://www.saphyre.eu/publications/. 
[9] E. A. Jorswieck, L. Badia, T. Fahldieck, E. Karipidis, and J. Luo, "Spectrum sharing improves the network efficiency for cellular operators," IEEE Commun. Mag., 2013, accepted for publication.

[10] M. Sternad, T. Ottosson, A. Ahlen, and A. Svensson, "Attaining both coverage and high spectral efficiency with adaptive OFDM downlinks," in Proc. IEEE Veh. Tech. Conf. (VTC), vol. 4, Oct. 2003, pp. 24862490 .

[11] FFR, "Interference mitigation considerations and results on frequency reuse," Siemens, Tech. Rep. 3GPP, TSG-RAN R1-050738, Sep. 2005.

[12] SFR, "Soft frequency reuse scheme for UTRAN LTE," Huawei, Tech. Rep. 3GPP, TSG-RAN R1-050507, May 2005.

[13] A. Paulraj, D. Gore, R. Nabar, and H. Bolcskei, "An overview of MIMO communications - a key to Gigabit wireless," Proc. IEEE, vol. 92, no. 2, pp. 198-218, Feb. 2004.

[14] E. Björnson, M. Kountouris, M. Bengtsson, and B. Ottersten, "Receive combining vs. multi-stream multiplexing in downlink systems with multi-antenna users," IEEE Trans. Signal Process., vol. 61, no. 13, pp. 3431-3446, Jul. 2013.

[15] D. Lee, H. Seo, B. Clerckx, E. Hardouin, D. Mazzarese, S. Nagata, and K. Sayana, "Coordinated multipoint transmission and reception in LTE-advanced: Deployment scenarios and operational challenges," IEEE Commun. Mag., vol. 50, no. 2, pp. 148-155, Feb. 2012.

[16] J. Mitola III, "Cognitive radio: An integrated agent architecture for software defined radio," Ph.D. dissertation, Royal Institute of Technology (KTH), Sweden, 2000.

[17] A. Goldsmith, S. Jafar, I. Marić, and S. Srinivasa, "Breaking spectrum gridlock with cognitive radios: An information theoretic perspective," Proc. IEEE, vol. 97, no. 5, pp. 894-914, May 2009.

[18] V. Valenta, R. Maršálek, G. Baudoin, M. Villegas, M. Suarez, and F. Robert, "Survey on spectrum utilization in Europe: Measurements, analyses and observations," in Proc. Int. Conf. on Cognitive Radio Oriented Wireless Networks and Commun. (CrownCom), Cannes, France, Jun. 2010, pp. 1-5. 
[19] T. Marzetta, "Noncooperative cellular wireless with unlimited numbers of base station antennas," IEEE Trans. Wireless Commun., vol. 9, no. 11, pp. 3590-3600, Sep. 2010.

[20] F. Rusek, D. Persson, B. K. Lau, E. Larsson, T. Marzetta, O. Edfors, and F. Tufvesson, "Scaling up MIMO: Opportunities and challenges with very large arrays," IEEE Signal Process. Mag., vol. 30, no. 1, pp. 40-60, Jan. 2013.

[21] X. Wu, B. Murherjee, and D. Ghosal, "Hierarchical architectures in the third-generation cellular network," IEEE Wireless Commun. Mag., vol. 11, no. 3, pp. 62-71, Jun. 2004.

[22] A. Lozano, R. Heath, and J. Andrews, "Fundamental limits of cooperation," IEEE Trans. Inf. Theory, vol. 59, no. 9, pp. 5213-5226, Sep. 2013.

[23] W. Utschick and J. Brehmer, "Monotonic optimization framework for coordinated beamforming in multicell networks," IEEE Trans. Signal Process., vol. 60, no. 4, pp. 1899-1909, Apr. 2012.

[24] Z.-Q. Luo and S. Zhang, "Dynamic spectrum management: complexity and duality," IEEE J. Sel. Topics Signal Process., vol. 2, no. 1, pp. 57-73, Feb. 2008.

[25] C. E. Shannon, "Two-way communication channels," in Proc. 4th Berkeley Symp. Math. Statist. Probab., vol. I, Berkeley, CA, 1961, pp. 611-644.

[26] E. C. van der Muelen, "The discrete memoryless channel with two senders and one transmitter," in Proc. IEEE Int. Symp. on Inf. Theory (ISIT), Tsahkadsor, Armenian SSR, 1971, pp. 103-135.

[27] R. Ahlswede, "Multiway communication channels," in Proc. IEEE Int. Symp. on Inf. Theory (ISIT), Tsahkadsor, Armenian SSR, 1971, pp. $23-52$.

[28] H. H. J. Liao, "Multiple access channels," Ph.D. dissertation, University of Hawaii, Honolulu, HI, 1972.

[29] A. D. Wyner, "Recent results in the Shannon theory," IEEE Trans. Inf. Theory, vol. 20, no. 1, pp. 2-10, Jan. 1974.

[30] T. M. Cover, "An achievable rate region for the broadcast channel," IEEE Trans. Inf. Theory, vol. 21, no. 4, pp. 399-404, Jul. 1975. 
[31] R. S. Cheng and S. Verdú, "Gaussian multiacccess channels with ISI: Capacity region and multiuser water-filling," IEEE Trans. Inf. Theory, vol. 39, no. 3, pp. 773-785, May 1993.

[32] W. Yu and J. Cioffi, "Sum capacity of gaussian vector broadcast channels," IEEE Trans. Inf. Theory, vol. 50, no. 9, pp. 1875-1892, Sep. 2004 .

[33] I. E. Telatar, "Capacity of multi-antenna Gaussian channels," European Trans. on Telecommun., vol. 10, no. 6, pp. 585-595, Nov./Dec. 1999.

[34] A. El Gamal and T. M. Cover, "Multiple user information theory," Proc. IEEE, vol. 68, no. 12, pp. 1466-1483, Dec. 1980.

[35] A. El Gamal and Y.-H. Kim, Network Information Theory. Cambridge University Press, 2011.

[36] T. M. Cover, "Broadcast channels," IEEE Trans. Inf. Theory, vol. 18, no. 1, pp. 2-14, Jan. 1972.

[37] P. P. Bergmans, "Random coding theorem for broadcast channels with degraded components," IEEE Trans. Inf. Theory, vol. 19, no. 2, pp. 197-207, Mar. 1973.

[38] R. G. Gallager, "Capacity and coding for degraded broadcast channels," Probl. Inf. Transm., vol. 10, no. 3, pp. 3-14, Mar. 1974.

[39] G. Caire and S. Shamai, "On the achievable throughput in the multiple antenna Gaussian broadcast channel," IEEE Trans. Inf. Theory, vol. 49, no. 7, pp. 1691-1706, Jul. 2003.

[40] S. Viswanath, N. Jindal, and A. Goldsmith, "Duality, achievable rates, and sum-rate capacity of Gaussian MIMO broadcast channels," IEEE Trans. Inf. Theory, vol. 49, no. 10, pp. 2658-2668, Oct. 2003.

[41] P. Viswanath and D. Tse, "Sum capacity of the vector Gaussian broadcast channel and uplink-downlink duality," IEEE Trans. Inf. Theory, vol. 49, no. 8, pp. 1912-1921, Aug. 2003.

[42] W. Yu, W. Rhee, S. Boyd, and J. M. Cioffi, "Iterative water-filling for Gaussian vector multiple-access channels." IEEE Trans. Inf. Theory, vol. 50, no. 1, pp. 145-152, Jan. 2004. 
[43] H. Weingarten, Y. Steinberg, , and S. Shamai, "The capacity region of the Gaussian multiple-input multiple-output broadcast channel," IEEE Trans. Inf. Theory, vol. 52, no. 9, pp. 3936-3964, Sep. 2006.

[44] M. H. M. Costa, "Writing on dirty paper," IEEE Trans. Inf. Theory, vol. 29, no. 3, pp. 439-441, May 1983.

[45] R. Ahslwede, "The capacity region of a channel with two senders and two receivers," Ann. Prob., vol. 2, pp. 805-814, Oct. 1974.

[46] T. Han and K. Kobayashi, "A new achievable rate region for the interference channel," IEEE Trans. Inf. Theory, vol. 27, no. 1, pp. 49-60, Jan. 1981.

[47] H. Sato, "The capacity of the Gaussian interference channel under strong interference," IEEE Trans. Inf. Theory, vol. 27, no. 6, pp. 786788, Jun. 1981.

[48] V. S. Annapureddy and V. V. Veeravalli, "Gaussian interference networks: Sum capacity in the low interference regime and new outer bounds on the capacity region," IEEE Trans. Inf. Theory, vol. 55, no. 7, pp. 3032-3050, Jul. 2009.

[49] G. Kramer, "Outer bounds on the capacity of Gaussian interference channels," IEEE Trans. Inf. Theory, vol. 50, no. 3, pp. 581-586, Mar. 2004 .

[50] R. Etkin, D. Tse, and H. Wang, "Gaussian interference channel capacity to within one bit," IEEE Trans. Inf. Theory, vol. 54, no. 12, pp. 55345562, Dec. 2008.

[51] V. Cadambe and S. A. Jafar, "Interference alignment and the degrees of freedom for the $k$-user interference channel," IEEE Trans. Inf. Theory, vol. 54, no. 8, pp. 3425-3441, Aug. 2008.

[52] S. Karmakar and M. K. Varanasi, "The capacity region of the MIMO interference channel and its reciprocity to within a constant gap," IEEE Trans. Inf. Theory, vol. 49, no. 8, pp. 4781-4797, Aug. 2013.

[53] E. A. Jorswieck, E. G. Larsson, and D. Danev, "Complete characterization of the Pareto boundary for the MISO interference channel," IEEE Trans. Signal Process., vol. 56, no. 10, pp. 5292-5296, Oct. 2008. 
[54] K. M. Ho, D. Gesbert, E. Jorswieck, and R. Mochaourab, "Beamforming on the MISO interference channel with multi-user decoding capability," IEEE Trans. Inf. Theory, 2011, submitted. Available: http://arxiv.org/abs/1107.0416.

[55] — - "Beamforming on the MISO interference channel with multi-user decoding capability," in Proc. IEEE Asilomar Conf. Signals, Systems, and Computers, Pacific Grove, CA, Nov 2010, pp. 1196-1201.

[56] R. Mochaourab and E. A. Jorswieck, "Robust beamforming in interference channels with imperfect transmitter channel information," Signal Processing, vol. 92, no. 10, pp. 2509-2518, Oct. 2012.

[57] L. Li and A. J. Goldsmith, "Capacity and optimal resource allocation for fading broadcast channels-Part II: Outage capacity," IEEE Trans. Inf. Theory, vol. 47, pp. 1103-1127, Mar. 2001.

[58] L. Li, N. Jindal, and A. J. Goldsmith, "Outage capacities and optimal power allocation for fading multiple-access channels," IEEE Trans. Inf. Theory, vol. 51, pp. 1326-1347, Apr. 2005.

[59] E. Karipidis, A. Gründinger, J. Lindblom, and E. G. Larsson, "Paretooptimal beamforming for the MISO interference channel with partial CSI," in Proc. IEEE Int. Workshop on Computational Advances in Multi-Sensor Adaptive Proces. (CAMSAP), Aruba, Dec. 2009, pp. 13 16 , invited.

[60] R. Mochaourab and E. A. Jorswieck, "Optimal beamforming in interference networks with perfect local channel information," IEEE Trans. Signal Process., vol. 59, no. 3, pp. 1128-1141, Mar. 2011.

[61] R. Zhang and S. Cui, "Cooperative interference management in multicell downlink beamforming," IEEE Trans. Signal Process., vol. 58, no. 10 , pp. 5450-5458, Oct. 2010.

[62] R. Mochaourab and E. Jorswieck, "Exchange economy in two-user multiple-input single-output interference channels," IEEE J. Sel. Topics Signal Process., vol. 6, no. 2, pp. 151-164, Apr. 2012.

[63] Q. Shi, M. Razaviyayn, Z.-Q. Luo, and C. He, "An iteratively weighted MMSE approach to distributed sum-utility maximization for a MIMO interfering broadcast channel," IEEE Trans. Signal Process., vol. 59, no. 9, pp. 4331-4340, Sep. 2011. 


\section{Papers}

The articles associated with this thesis have been removed for copyright reasons. For more details about these see:

http://urn.kb.se/resolve?urn=urn:nbn:se:liu:diva-100820 